This item was submitted to Loughborough's Research Repository by the author.

Items in Figshare are protected by copyright, with all rights reserved, unless otherwise indicated.

\title{
Linear friction weld process monitoring of fixture cassette deformations using empirical mode decomposition
}

PLEASE CITE THE PUBLISHED VERSION

http://dx.doi.org/10.1016/j.ymssp.2015.02.005

\section{PUBLISHER}

(C) Elsevier Ltd.

\section{VERSION}

AM (Accepted Manuscript)

\section{PUBLISHER STATEMENT}

This work is made available according to the conditions of the Creative Commons Attribution-NonCommercialNoDerivatives 4.0 International (CC BY-NC-ND 4.0) licence. Full details of this licence are available at: https://creativecommons.org/licenses/by-nc-nd/4.0/

\section{LICENCE}

CC BY-NC-ND 4.0

\section{REPOSITORY RECORD}

Bakker, O.J., C. Gibson, P. Wilson, Niels Lohse, and A.A. Popov. 2019. "Linear Friction Weld Process Monitoring of Fixture Cassette Deformations Using Empirical Mode Decomposition". figshare. https://hdl.handle.net/2134/17439. 


\title{
Linear friction weld process monitoring of fixture cassette deformations using empirical mode decomposition
}

\author{
O.J. Bakker ${ }^{1 \mathrm{a}}$, C. Gibson ${ }^{\mathrm{b}}$, P. Wilson ${ }^{\mathrm{b}}$, N. Lohse ${ }^{2 \mathrm{a}}$, A.A. Popov ${ }^{\mathrm{a}}$ \\ ${ }^{a}$ Manufacturing and Process Technologies Research Division, Faculty of Engineering, University of Nottingham, University Park, Nottingham, \\ $N G 72 R D, U K$ \\ ${ }^{b}$ Rolls-Royce plc, PO Box 31, Derby, DE24 8BJ, UK
}

\begin{abstract}
Due to its inherent advantages, linear friction welding is a solid-state joining process of increasing importance to the aerospace, automotive, medical and power generation equipment industries. Tangential oscillations and forge stroke during the burn-off phase of the joining process introduce essential dynamic forces, which can also be detrimental to the welding process. Since burn-off is a critical phase in the manufacturing stage, process monitoring is fundamental for quality and stability control purposes. This study aims to improve workholding stability through the analysis of fixture cassette deformations. Methods and procedures for process monitoring are developed and implemented in a fail-or-pass assessment system for fixture cassette deformations during the burn-off phase. Additionally, the denoised signals are compared to results from previous production runs. The observed deformations as a consequence of the forces acting on the fixture cassette are measured directly during the welding process. Data on the linear friction-welding machine are acquired and de-noised using empirical mode decomposition, before the burn-off phase is extracted. This approach enables a direct, objective comparison of the signal features with trends from previous successful welds. The capacity of the whole process monitoring system is validated and demonstrated through the analysis of a large number of signals obtained from welding experiments.
\end{abstract}

Keywords: linear friction welding, process condition monitoring, Hilbert-Huang transform, time-frequency analysis, empirical mode decomposition, non-stationary signal

\section{Introduction}

This paper discusses the setting up of a condition monitoring system to verify the workholding stability during a linear friction welding process using empirical mode decomposition. The Introduction is split into four parts. Firstly, the background of the work is discussed in Section 1.1. After which in Section 1.2 a review is held of closely related work on condition monitoring systems found in the literature. Subsequently, some relevant developments of empirical mode decomposition for manufacturing process monitoring purposes are discussed in Section 1.3. Finally, the outline of the rest of the paper is presented in Section 1.4.

\subsection{Background: Linear Friction Welding}

The linear friction welding process has many advantages and benefits compared with other joining processes. From a technical point of view, it has the advantage that it can be used to join two complex shaped parts. The economical benefits of the process are that the process is fast and the welding area requires little preparation. Additionally, it needs relatively low energy input and requires no consumables as means to assist the welding process, nor is there an emission of dangerous substances during the process, making it a relatively sustainable process.

However, a considerable perceived drawback of the process is that for most applications the weld quality cannot be inspected with appropriate non-destructive testing methods. Hence the weld verification is done by means of

\footnotetext{
${ }^{1}$ Corresponding author: Tel.: +44 11574 84709; E-mail address: ottojan. bakker@nottingham.ac.uk

${ }^{2}$ Currently working for the School of Mechanical and Manufacturing Engineering, Loughborough University, Loughborough, Leicestershire, LE11 3TU, UK
} 


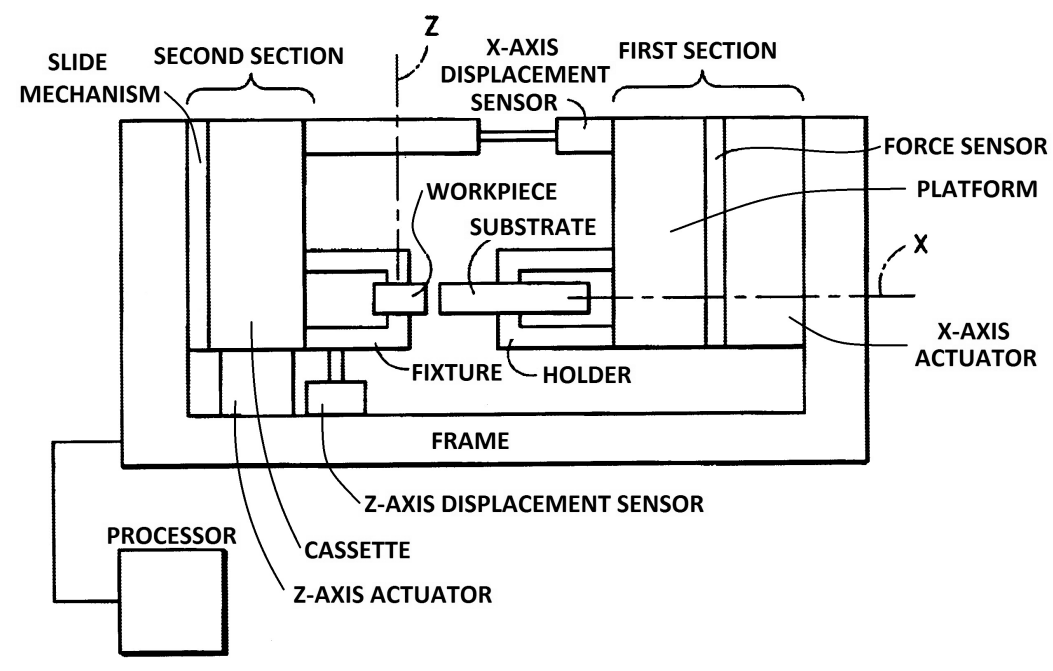

Figure 1: Schematics of a linear friction welding machine. Adapted from Ref. [2, Fig. 1]

process condition monitoring in combination with statistical process monitoring, to ensure a repeatable production of reliable welds. The outcome of the linear friction welding process depends on a set of controllable input parameters and other process parameters which can be monitored for process condition monitoring purposes. The most popular application of linear friction welding is the manufacture of "blisks" (bladed disks) for aero-engines. Blisks have the advantage over traditionally designed disk assemblies that the fir tree joint is no longer needed, which leads to up to $30 \%$ weight reduction and improvements in aerodynamic efficiency [1]. The work presented in this paper focusses on the monitoring of the workholding stability during the burn-off phase.

In Refs $[2,3]$ the basics of the working of a linear friction welding machine for blisk manufacturing are explained. An adapted version of the working diagram that can be found in Ref. [2] is shown in Fig. 1. As can be seen in the figure, generally a LFW machine consists of a frame and two sections. The first section holds the substrate (disk) on a platform. An actuator can move this platform in a controlled manner in the forging direction $x$. The second section is used to provide the reciprocal oscillatory tangential motion required for the LFW process. It comprises of an actuator that provides the motion to drive the cassette in the tangential direction $z$. The machine's tooling consists of the inner cage wherein a cassette is placed that holds the workpiece (blade) [4]. To ensure workholding stability in the $y$-direction, the cassette deformation during the process should be kept below certain limits.

During the LFW process the workpiece and substrate are datumed and brought in contact with each other up to a certain contact pressure. This is also known as the contact phase [3] and is depicted in Fig. 2. The workpiece in Fig. 1 is moved in a linear, reciprocating way, tangential and relative to the other part. The friction generates heat, which is enough to make the material ductile. In the first instance the rubbing is used to remove the outer layer of material, this is called burn-off in this work and comprises what is generally known as the initial (conditioning, [3]), the transition and equilibrium (frictional, [3]) and the first part of the deceleration (forge, [3]) phases [5], see Fig. 2. In the ramp-down phase, which is the first stage of the deceleration phase in Ref. [5] the amplitude of the tangential reciprocating motion is decreased, as can be seen in Fig. 2. After this, when the reciprocating movement is completely stopped, and the moving part is placed in the desired position, the part and substrate are then forged onto each other in the second part of the forge or deceleration phase. It should be noted that in the work presented here, the welding process is monitored for the duration of the tangential motion. These oscillations take place from the initial phase to the ramp-down phase, as shown in Fig. 2 and this period is denoted as 'period of interest' in the figure. Furthermore, it should be noted that this work studies the reproducibility of the welds, hence, the analysis of the influence of the different phases on the weld is not a part of this study. The interested reader is referred to the papers by Vairis and 


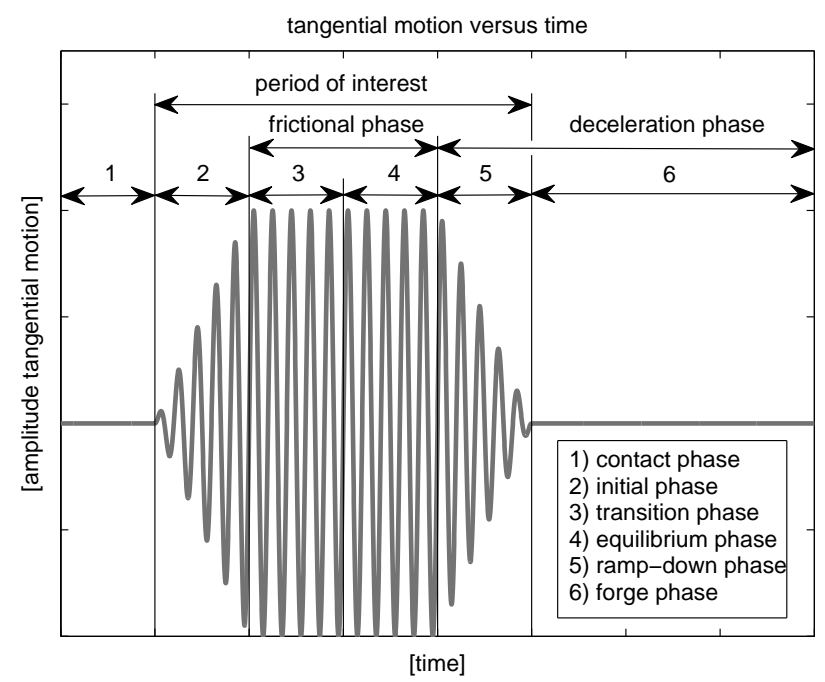

Figure 2: Weld phases and period of interest, naming conventions taken from Refs [3-5].

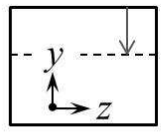

compression

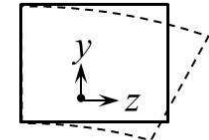

bending

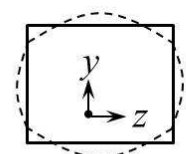

barrelling

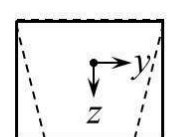

keystoning

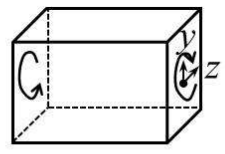

warping

Figure 3: Definition of the cassette deformations.

Frost [5] and Bhamji et al. [3], where the influence of the weld phases on the parameters is discussed in much greater detail. However, the methodology presented here can be easily extended to do such a mechanistic analysis of the weld parameters.

A LFW machine is typically equipped with several sensors that can be used to monitor the process variables, some of these sensor are shown in Fig. 1. The processor is used to control the processes and capture the data from the sensory system. On the linear friction welding machine used for the experiments presented in this work, force sensors are placed on the cassette by the machine builder for the purpose of stable workholding, using these variables an indirect analysis can be made of the workholding stability. When all the measured forces are included in the analysis, such that a proper force balance can be established, inverse mechanics can be utilized, if necessary, to calculate whether the required conditions for workholding are met, similar to the scheme proposed by Hameed et al. [6] to obtain the cutting forces from the reaction forces on fixture locators. However for the purposes here, ultimately aimed at supporting the application of linear friction welding in production of components for aero-engines, the interest lays in monitoring the weld and compare it with other welds.

The force sensors are utilized to monitor the following deformations shown in Fig. 3: compression, bending, barrelling, keystoning and warping, which, hence, are related to the workholding stability in $y$-direction, the $x, y, z$-directions of the right-handed coordinate system are given in each of the deformation modes shown in Fig. 3.

\subsection{Manufacturing process monitoring}

Manufacturing process monitoring can be done with a variety of sensors. Typically, the sensor output is a discrete signal in the time domain. When necessary, the signal can be transformed to the frequency domain. Over the 
last two or three decades, manufacturing process monitoring for quality control has received much attention in the manufacturing research community [7-11].

When focussing more specifically on area of monitoring systems for machining operations, according to Refs $[8,11]$, manufacturing process monitoring can be classified in four broad approaches within a process monitoring application or scope. These scopes are e.g. tool conditions, process condition, machine tool state or surface roughness and dimensional variations. The four approaches within each of these applications are: (1) model-based, (2) experimental, (3) design of experiment, (4) artificial intelligence. For the purposes of LFW process monitoring, a brief review of the state-of-the-art in tool condition monitoring and process condition \& machine tool state is conducted in Sections 1.2.1 and 1.2.2 respectively.

\subsubsection{Tool condition monitoring}

1. As tool wear and tool breakage are the outcome of highly random and stochastic processes, it is currently impossible to establish reliable models that predict the tool life time or model the occurrence of tool breakage. Note that tool breakage can cause severe disruption to the production process and should be avoided if possible. For this reason, there is a strong emphasis on the prediction of tool life time and or tool breakage, such that the cutting tool can be replaced on time.

2. Tansel et al. [12] investigated the impedance, response to excitation and the propagation of Lamb waves on the tool surface to estimate the wear of drill bits.

Bisu et al. [13] executed a spectral analysis for machine tool diagnosis and tool condition monitoring and did further data processing with the Hilbert transform and spectral envelope analysis (Short-Time Fourier Transform (STFT)) to obtain a waterfall-type diagram to study the behaviour of the frequency spectrum over the elapse of time to enhance their spectral analysis. Kalvoda and Hwang [14] applied the Hilbert-Huang transform (HHT) to the measured signal of monitor tool wear in the frequency domain and compared the outcome of the results with the widely applied Fourier transform.

Shao et al. [15] applied a modified single-channel blind sources separation (BSS) technique based on the wavelet transform and independent component analysis to separate the source signals related to a milling cutter and a spindle for the application of tool breakage monitoring. Chen et al. [16] measure the tool vibrations, apply the wavelet transform and use a logistical correlation study of the wavelet energy is made to identify feature frequency bands that indicate tool wear.

3. Sivasakthivel et al. [17] conducted a series of experiments to apply the response surface methodology to predict the vibration amplitude for the following cutting parameters: the helix angle of cutting tool, the spindle speed, the feed rate, and the axial and radial depth of cut. Chen et al. [18] applied a singular spectrum analysis to relate surface roughness and tool vibration to the spindle speed, feed rate, cutting depth, cutting feed direction and holder type.

4. Yang and Yu [19] built a grinding wheel monitoring system to predict grinding wheel wear based on a wavelet analysis of the acoustic emissions from the grinding process feeding into a support vector machine algorithm. Boutros and Liang [20] applied hidden Markov models for the detection and diagnosis of faults in bearing and cutting tool systems.

Kilundu et al. [21] studied tool wear by extracting features associated with tool wear from three different frequency bands, applying a windowed singular spectrum analysis and several machine learning techniques. Tobon-Mejia et al. [22] measured cutting forces, acoustic emissions and vibrations to train a 'mixture of Gaussians Hidden Markov Model' to build a model to predict the useful lifetime of a milling cutter. Yen et al. [23] trained a neural network $(\mathrm{NN})$ with the frequency spectrum of acoustic emissions and applied a self-organization feature map to make the method more robust with respect to influence of noise.

\subsubsection{Process and machine tool conditions}

1. Trimble et al. [24] established a finite element model that predicts tool forces for different friction stir welding parameters and tool failure. Their model has been validated by experimental results. Kalinski and Galewski [25] built a mechanical model to simulate the cutting for a ball-end milling process and applied optimal-linear spindle speed control to prevent the occurrence of chatter in the cutting process.

Zhou et al. [26] monitor an aspect of the machine tool state: the fault in a machine tool feed-axis gearbox utilizing the built-in position sensors. The signal is split in frequency bands using the ensemble empirical 
mode decomposition (EEMD), which overcomes the mode mixing occurring in the standard empirical mode decomposition method (EMD).

2. Pérez-Canales et al. [27] calculated the approximate entropy (ApEn) in the measured vibration to identify chatter conditions in the signal and compared it with a Fourier analysis of the same signals.

Jin and Shi [28] applied a wavelet analysis to check for errors in the tonnage signal of a stamping process.

Longangbach and Kurfess [29] designed a gauge that can measure the diameter of a workpiece in-process on a turning lathe using LVDTs (linear variable distance transducers).

3. Rabani et al. [30] conducted a series of experiments to link the transfer rate of energy to the input jet energy, area of abraded footprint and jet feed velocity for monitoring of abrasive waterjet milling process.

4. Kong and Nahavandi [31] established a control system using NN, inputing force signals and acoustic emissions, to monitor the forging process and predict tool life.

\subsubsection{Discussion of Related Work}

Even from this short review, it is abundantly clear that a plethora of techniques is applied for the purpose of condition monitoring, as each application is unique or has unique aspects to it. Secondly, (combinations of) certain techniques have certain advantages and disadvantages which can be employed for the monitoring problem and are sometimes used because of the previous experience or familiarity. Also using these techniques results in a working solution because of constraints of a non-technical nature. This is related to another important observation that should be made when reviewing the relevant literature [7-11]: the large majority of the papers that can be found describe work that has been carried out in laboratories, but there are almost no commercially available condition monitoring systems for manufacturing applications. This is in part because of the above mentioned uniqueness of each different application and also because there is still a low level of standardization which hampers the automated integration of sensors in a monitoring systems, establishing of learning models and automated diagnostics of the monitored process or equipment. Furthermore, the majority of the monitoring considers the analysis of stationary signals, whereas most variables in a production system are inherently non-stationary. However, as tools for the analysis of non-stationary signals become more widely available, more recent works do analyze non-stationary signals, e.g. Refs [14, 15, 26, 28].

\subsection{Hilbert-Huang Transform}

The Hilbert-Huang transform was established in 1998 by Huang et al. [32] to analyze nonlinear and non-stationary data. This uses the empirical mode decomposition technique to divide a signal in a number of components, called intrinsic mode functions (IMF), that are in different frequency bands. The mode decomposition does not work flawlessly. One of the major issues is mode mixing. When this phenomenon occurs, the frequency bands are smeared out over multiple modes. As a result, the signal analysis can become problematic. To overcome this problem, several improvements and modifications have been suggested. The two most prominent methods encountered in applications are the ensemble empirical mode decomposition and the combination of wavelet packet decomposition and the Hilbert-Huang transform. Wu and Huang [33] proposed the ensemble empirical mode decomposition that deals with the white noise that causes mode mixing. Peng et al. [34] proposed the application of the wavelet packet transform to split the signal into a set of various narrow band signals before the use of the empirical mode decomposition.

Besides the field of machine monitoring, the Hilbert-Huang Transform and empirical mode analysis have been successfully applied for structural health monitoring [35], fault monitoring in rotating machinery [36], (spindle) bearings [37] and gearboxes [26].

\subsection{Paper outline}

For the purpose of monitoring the forces on the cassette in the LFW process, this paper follows the methodology typically applied to establish a monitoring system for a manufacturing process. In Section 2, firstly the applied methodology is explained in Section 2.1. Then, the signal de-noising and further processing are discussed in Section 2.2. After which the extraction of the forces during the burn-off phase from the whole measured force vectors is discussed in Section 2.3. A series of welding experiments have been conducted by Rolls-Royce plc. The methodology presented in this paper has been applied to the measured forces for these experiments. In Section 3 the trends for de-noised forces are discussed, followed by the conclusions that can be drawn from this work in Section 4. 


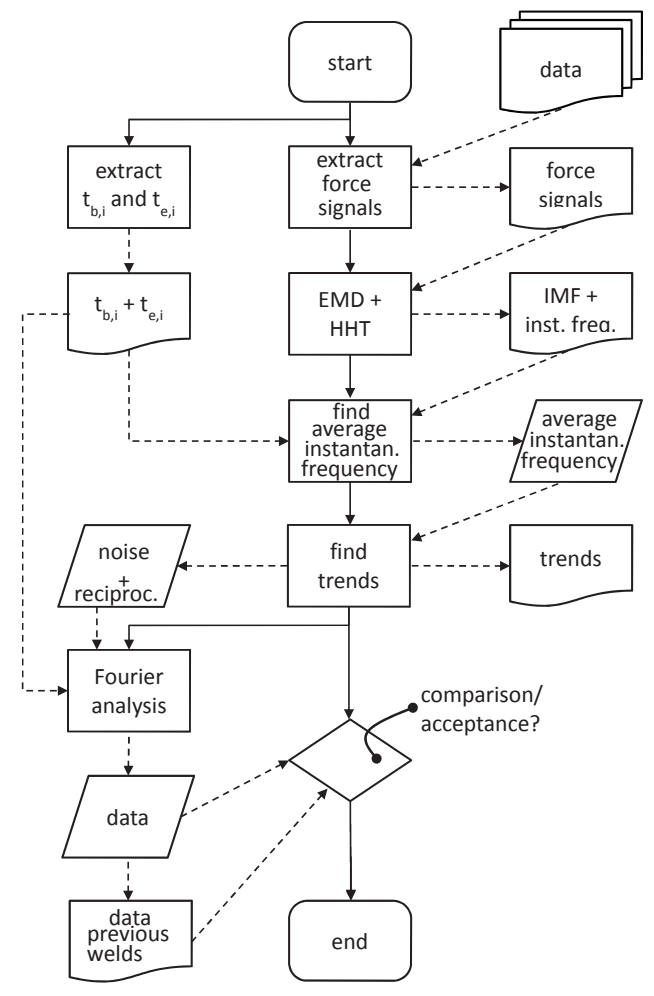

Figure 4: Software flowchart.

\section{Method}

\subsection{Applied methodology}

The basic methodology to design monitoring and decision making systems is well established, see e.g. the six key areas examined in the review paper by Abellan-Nebot and Subirón [7]. However, each time the methodology needs to be tailored to suit an application, and the right tools need to be applied. The flowchart of the methodology to develop the cassette forces monitoring system is shown in Fig. 4.

As one can see in Fig. 4, firstly, the force measurement vectors are collected from the sensory system of the LFW machine. Subsequently, these vectors are de-noised and the burn-off period is extracted from the signal. After which the welding trend is compared against the values of previous successful production runs.

Apart from comparing the forces with previous production runs for the purpose of process condition monitoring, a secondary objective for the process monitoring system is to detect other oscillatory content in the measured signals. As the burn-off phase consists of the oscillatory motion that occurs during a relatively short phase within the whole LFW process - usually between 1 and $2 \mathrm{~s}$, depending on the application - it is easy to extract this period as explained later in Section 2.3.

\subsection{Signal de-noising}

\subsubsection{Effects of filtering}

The combination of noise, forced vibrations and tangential oscillations occurring in the LFW machine can be traced in the measured forces acting on the cassette. When the signal is differentiated with respect to the time, these phenomena can obscure the trend of the force rate $\left(\frac{\mathrm{d}}{\mathrm{d} t}\right)$ and the change of force rate $\left(\frac{\mathrm{d}^{2}}{\mathrm{~d} t^{2}}\right)$. For this reason, some filtering of the signal is essential, especially, for removing the oscillatory component introduced by the reciprocating movement of the LFW machine. However, the filtering action introduces extra distortion to the signal. In Fig. 5 the 
effects of several filtering strategies on the trend in one of the force signals are shown. As the reciprocal frequency $\omega_{\mathrm{r}}$ is the dominant frequency, the first choice is to apply a notch filter (a band-stop filter with a narrow stopband) with a notch frequency equal to the reciprocal frequency $\omega_{\mathrm{r}}$ of the LFW process. However, the signal contains higher harmonics, furthermore, applying purely a notch filter results in spurious harmonics as can be seen in the left hand detail in the top most sub-figure in Fig. 5. Consequently, the application of a notch filter does not give the desired results. The signal can be further smoothed by adding certain low-pass filtering action. Traditionally, to smooth the signal, only a low-pass filter is used. This means that it filters out the signal components from the high-frequency range. A Fourier transform shows that a steep change in the gradient of the measured signal contains many highfrequency components. Hence filtering with a typical low-pass smoothing filter will always change the gradient to make it less steep. This is shown in the centre sub-figure in Fig. 5, where the effect of filters with an increasingly shorter pass-band is shown. As can be seen in the figure, the changes in the gradient of the filtered trend become increasingly dull. A more recent yet firmly established method is to de-noise signals using the wavelet transform. This form of de-noising is suited for the analysis and de-noising of signals of non-linear processes such as the LFW process. As can be seen in the bottom sub-figure in Fig. 5, the result of wavelet de-noising is superior to low-pass and notch filtering. However, as is demonstrated in the centre right detail, small noise spikes or serrations are unavoidable. The reason for this lies in the nature of the signal representation in the wavelet domain. According to the wavelet analysis when a noiseless signal is decomposed into sub-bands, a signal without noise has a relatively sparse signature in these sub-bands. The standard de-noising functions make use of this fact, by removing all signal components which are smaller than certain threshold values from the sub-bands. Any noise component that is larger than the threshold values remains in the sub-band, causing noise spikes and serrations. These small noise spikes or serrations come out as large noise spikes when the derivative of the trend is studied, which forms a large drawback for the analysis of the trends in the measured cassette forces. All this means that one has to pay the price of a less accurate signal when applying filtering action to smooth the signal. When the smoothing filter is designed with a much too low cut-off frequency to remove the oscillatory component in the signal, the filter will cut out much of the undesired noise etc., but will significantly decrease the accuracy of the signal as the changes in the gradient become duller. Therefore, filter design for signal smoothing filters comprises an optimisation of the trade-off between the loss of accuracy in the gradients and the remaining noise content.

\subsubsection{EMD principle [32]}

The Hilbert-Huang transform (HHT) is devised to analyze nonlinear and non-stationary signals. The fundamental part of the HHT is the empirical mode decomposition (EMD). Any real-valued signal $x(t)$ can be separated into a set of intrinsic mode functions (IMFs) $c_{i}(t)$ and a residual $r(t)$ so that:

$$
x(t)=\sum_{i=1}^{n} c_{i}(t)+r_{n}(t) .
$$

Each individual IMF possesses its frequency band that can be analyzed. To obtain each IMF, the EMD algorithm runs until it finds an oscillatory function around the local mean defined by the upper and lower envelopes governed by the local maxima and minima, respectively. This oscillatory function is defined as follows:

1. The number of extrema differs from the number of zero-crossings in the IMF by zero or one at most.

2. At any point, the mean value of the lower and upper envelopes around the IMF is zero.

The EMD algorithm employs a sifting process, which works as follows. Firstly, it looks for the envelopes around the signal. Between these envelopes there is a mean $m_{1}$. The first component $h_{1}$ is formed by the difference between the data and the mean:

$$
h_{1}=x(t)-m_{1}
$$

In the consecutive sifting process, $h_{1}$ is used as data:

$$
h_{11}=h_{1}-m_{11},
$$

where the new component becomes $h_{11}$ and the new mean $m_{11}$. This sifting process is repeated $k$ times, until $h_{1 k}$ has converged close enough to the IMF criteria defined above. Then $h_{1 k}$ becomes

$$
h_{1 k}=h_{1(k-1)}-m_{1 k} \text {, }
$$




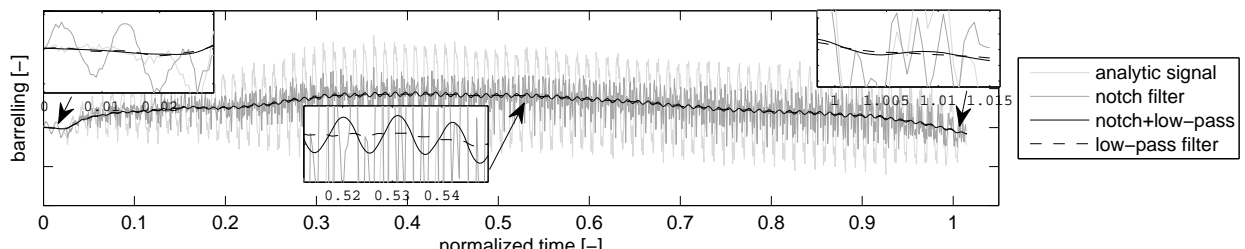

(a)
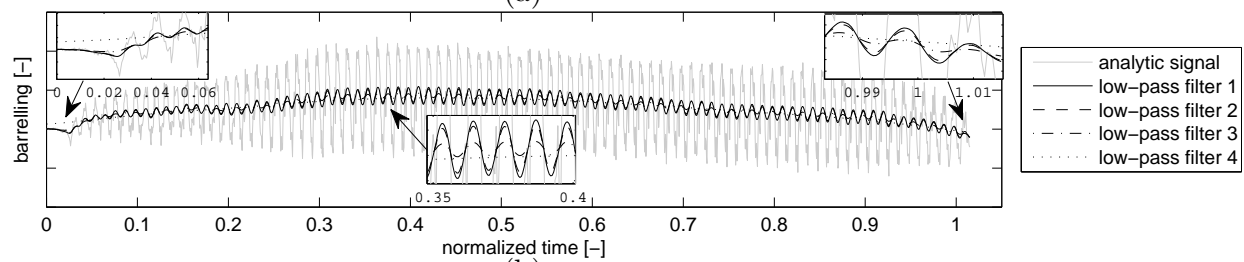

(b)

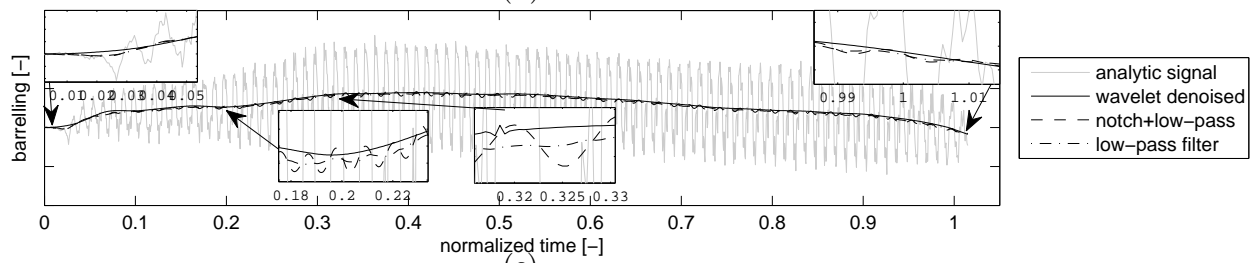

(c)

Figure 5: Effects of filtering and de-noising strategies. (a) The analytic signal compared with a filtered signals created by the application of a notch filter, a notch filter in combination with a low-pass filter and a low-pass filter only; (b) the analytic signal compared with several low-pass filter designs; (c) the analytic signal compared with wavelet de-noised and filtered signals. 
and $h_{1 k}$ is designated the first IMF, $c_{1}=h_{1 k}$. The first IMF contains all the shortest oscillations and hence possesses the highest frequency band in the signal. Subsequently, the first IMF is subtracted from the original signal as follows:

$$
r_{1}=x(t)-c_{1},
$$

where $r_{1}$ is the residual, which is then treated as data and the sifting procedure is repeated $n$ times until the values in residual $r_{n}$ are smaller than the threshold value of the sifting procedure stoppage criterion, or form a monotonic function. Eq. (5) is a crucial step in the EMD analysis carried out for this work. It allows the frequency bands to be studied individually and to be subtracted from the signal $x(t)$ to study the trend in the signal.

In the HHT, the instantaneous frequency is calculated using the Hilbert transform, which finds the complex conjugate $y(t)$ of any real valued function $x(t)$ as long as it is in the Lebesgue integrable function space [38], then:

$$
\mathcal{H}(t)=\frac{1}{\pi} \int_{+\infty}^{-\infty} \frac{x(\tau)}{t-\tau} \mathrm{d} t .
$$

The analytic signal is found by combining the real and the complex valued parts:

$$
z(t)=x(t)+i \mathcal{H}(t)=a(t) e^{i \theta(t)},
$$

where

$$
a(t)=\sqrt{x^{2}(t)+\mathcal{H}^{2}(t)}
$$

is the instantaneous amplitude of the signal and

$$
\theta(t)=\arctan \left(\frac{\mathcal{H}(t)}{x(t)}\right)
$$

is the instantaneous phase of the signal. Hence, the instantaneous frequency $\omega(t)$ simply becomes

$$
\omega(t)=\frac{\mathrm{d} \theta}{\mathrm{d} t} .
$$

\subsubsection{Frequency band selection}

In order to find the trend in the measured force signals, the IMFs containing the noise and oscillation need to be separated from the trend; the EMD process is used as a filter bank. The selection of the frequency bands is based on the physical meaning of its contents. This can be illustrated by analyzing the HHT of one of the force signals of the experimental welds presented in Section 3: barrelling forces, weld 7 in Table 2. The HHT consists of two parts: the IMFs and the instantaneous frequency. In Fig 6, all the IMFs for the duration of the burn-off phase of the barrelling force of weld 7 obtained with the EMD are shown. In this figure it can be seen that during the burn-off period the first six IMFs contain the noise and reciprocating motion induced oscillations. The corresponding instantaneous frequencies for each of the IMFs shown in Fig 6, are shown in Fig. 7. The instantaneous frequencies show that the IMFs are split up in frequency bands. Within the bandwidth of frequency bands the instantaneous frequency can move up or down over time. However, it can be seen that the frequency bands that comprise the trends contain frequencies lower than the reciprocating frequency $\omega_{\mathrm{r}}$, since they are lower than one in the plots in Fig. 7.

One-by-one experimental investigation using the HHT function in MATLAB ${ }^{\circledR}$ [39] of each of the signals reveals the same pattern, namely that the IMFs containing the trend of the measured force all have instantaneous frequencies lower than $\omega_{\mathrm{r}}$. Therefore it is possible to select the IMFs that form the noise and oscillation band by considering the average instantaneous frequency during the burn-off phase in an IMF which determines how many IMFs form the noise and oscillation band. Furthermore in Fig. 6b, it can be seen that IMF 7 is no longer recognisable as a typical IMF over the period of interest: there are no zero crossings here. Temporally the frequency in Fig $7 \mathrm{~b}$ of IMF 7 drops below zero, further indicating that the IMF locally doesn't meet Hilbert's criterion for instantaneous frequency. From Fig 7 it can be observed that the calculated instantaneous frequency is not a smooth line. The clearly visible dots indicate that sometimes the frequency is making a jump or even becomes negative. For the calculation of the value of the average instantaneous frequency, the results below zero are discarded. As a result, finding the average instantaneous frequency and separating the noise and oscillations from the trend form the third and forth step in the process, as is 


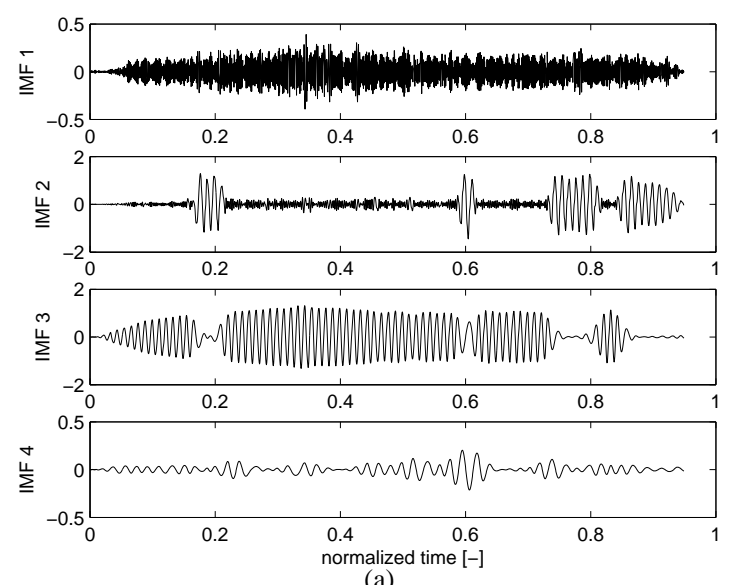

(a)

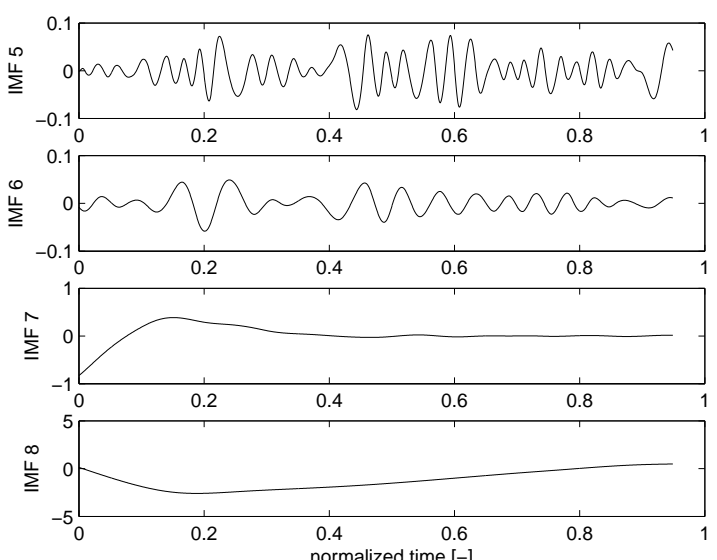

(b)
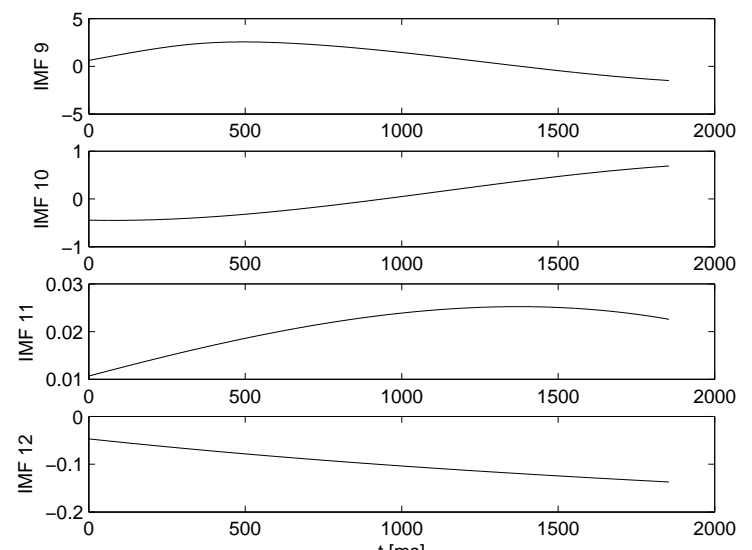

(c)

Figure 6: Intrinsic mode functions (IMFs) barrelling weld 7; burn-off period extracted from whole signal. (a) IMF 1-4, (b) IMF 5-8, (c) IMF 9-12. 

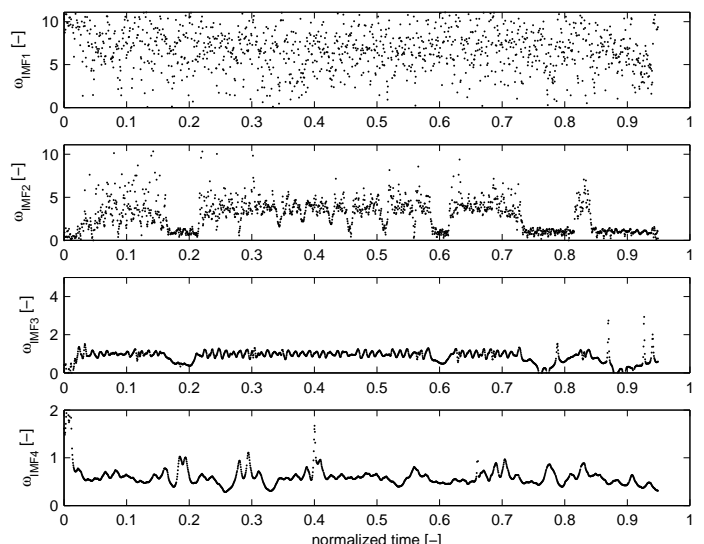

(a)
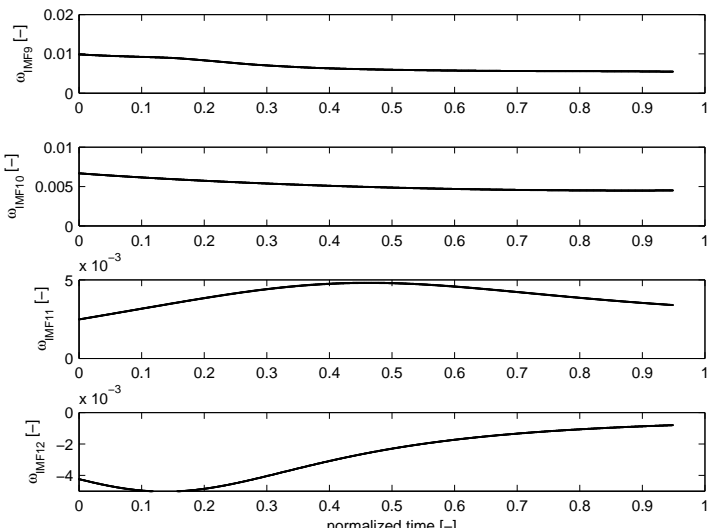

(c)
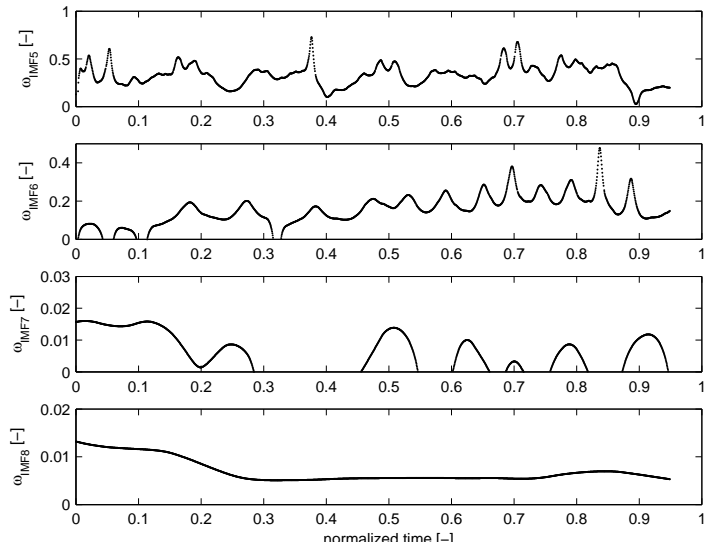

(b)

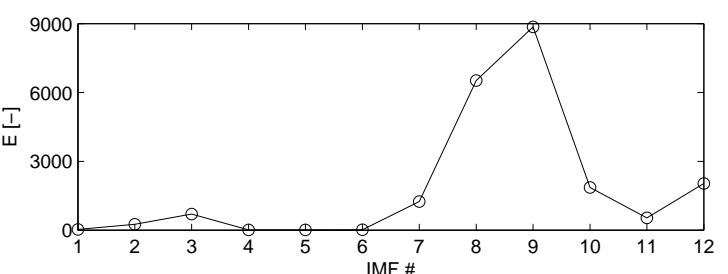

(d)

Figure 7: Instantaneous frequency per intrinsic mode function (IMF) barrelling weld 7, normalized with respect to the reciprocating frequency $\omega_{\mathrm{r}}$ and energy per IMF. (a) Instantaneous frequency IMF 1-4, (b) Instantaneous frequency IMF 5-8, (c) Instantaneous frequency IMF 9-12, (d) Energy per IMF. 


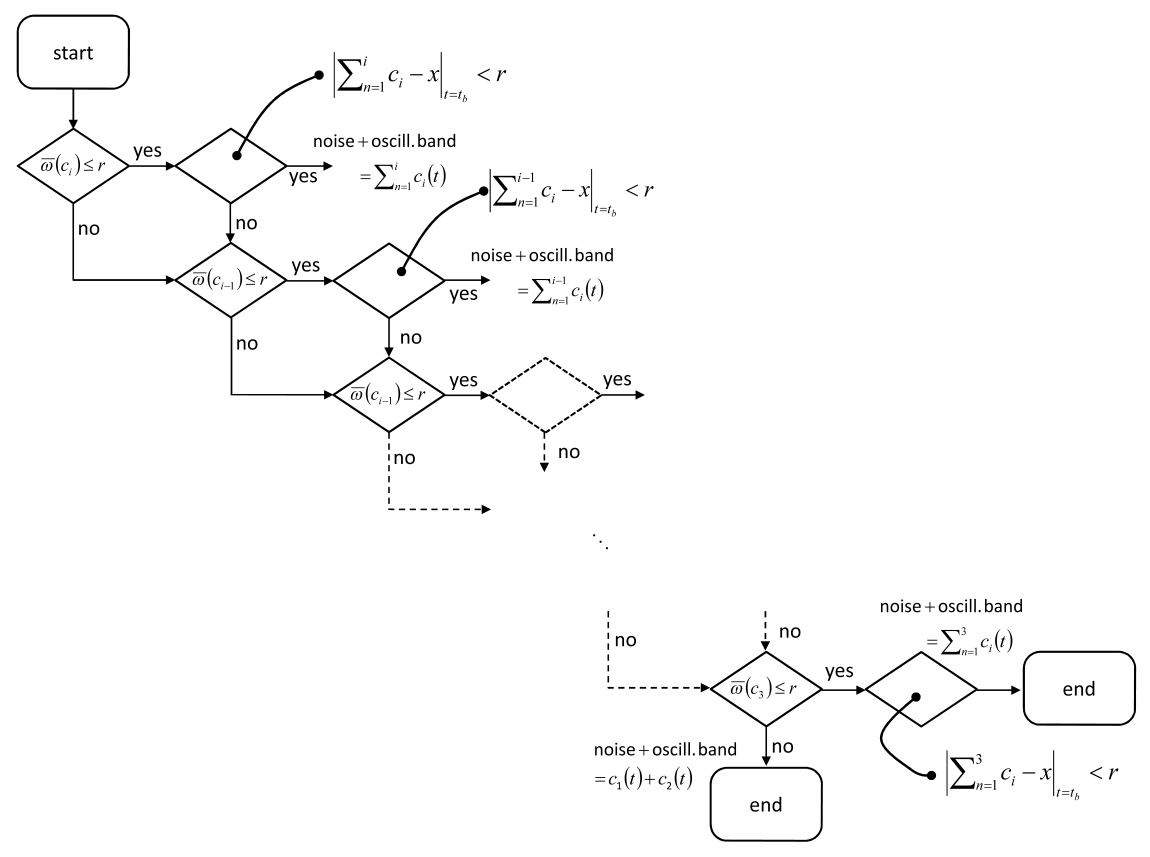

Figure 8: Algorithm to select number of IMFs to form noise and oscillation band and trend.

shown in Fig. 4. Note that the instantaneous frequency of IMF 12 in Fig. 7c is entirely below zero as this is residual and not a proper IMF; when investigating the instantaneous frequency over the whole time period from the beginning of the contact phase till the end of the forge phase, the frequency is zero, apart from the beginning and end and the period of motion along the $\mathrm{z}$-axis.

Beginning and end effects in the EMD process in the form of waves at the beginning and end of the signal which come in larger amplitudes and lower frequencies when the IMF number increases affect the accuracy of the trend. Therefore, after subtracting the IMFs that form the noise and oscillation bands, the beginning and end of the de-noised forces during the burn-off phase can significantly differ from the unprocessed measured signal. For this reason, the de-noised values at the beginnings and ends are compared with the unprocessed values and if the difference trespasses a certain value, one IMF is deducted from the noise and frequency band to make a better fit between the trend and the measured signal. As this is too detailed to show in Fig. 4 the pseudo algorithm for this step is shown in Fig. 8 and works as follows. Starting at the highest IMF number $n$ the algorithm checks if the average frequency $\bar{\omega}\left(c_{i}\right)$ of any IMF $c_{i}$ during the burn-off phase is higher than the reference value $r$. If the average frequency is below the the reference value, the algorithm will move one IMF number lower to $i-1$ and check whether the average frequency for this IMF higher than the reference value, if the average frequency is still larger than the reference value, the algorithm will move as many IMF numbers lower until the average frequency becomes smaller than the reference value, as can be seen in Fig. 8. If for a certain IMF the average frequency is below the reference value, the sum of all the IMFs 1 to $i$ should form the noise oscillation band. If the difference between the measured analytic signal and trend consisting of the sum of IMFs $i+1$ to $n$ at $t=t_{b}$ or $t=t_{e}$ is larger than a certain reference value $r$, the found trend is too inaccurate and IMF $c_{i}$ is subtracted from the noise and oscillation band and added to the trend. The difference between the measured signal and the new trend at at $t=t_{b}$ or $t=t_{e}$ again compared with reference value $r$. This procedure will repeat until the found difference is smaller than the reference value, as can be seen in Fig. 8.

Following experimental and automated analysis, it appears that, depending on which of the five forces is considered, band comprising the parts of the signal formed by the noise and oscillations is made up by the first $4-7$ IMFs containing the higher frequencies as can be seen in Table 1 .

If one considers the energy in the IMF, the spectral energy from the noise tends to decay for higher IMF numbers, which Flandrin et al. [40, Chapter 3] and Wu and Huang [40, Chapter 5] take as measure to establish a noise band con- 


\begin{tabular}{|c|c|c|c|c|c|}
\hline weld number & compression & bending & barrelling & keystoning & warping \\
\hline \hline 1 & $1-4$ & $1-8$ & $1-6$ & $1-6$ & $1-4$ \\
2 & $1-4$ & $1-7$ & $1-6$ & $1-6$ & $1-4$ \\
3 & $1-5$ & $1-8$ & $1-5$ & $1-6$ & $1-4$ \\
4 & $1-4$ & $1-8$ & $1-5$ & $1-5$ & $1-4$ \\
5 & $1-5$ & $1-8$ & $1-6$ & $1-5$ & $1-4$ \\
6 & $1-4$ & $1-6$ & $1-6$ & $1-6$ & $1-4$ \\
7 & $1-5$ & $1-8$ & $1-6$ & $1-6$ & $1-5$ \\
\hline
\end{tabular}

Table 1: Number of IMFs that form noise and oscillation.
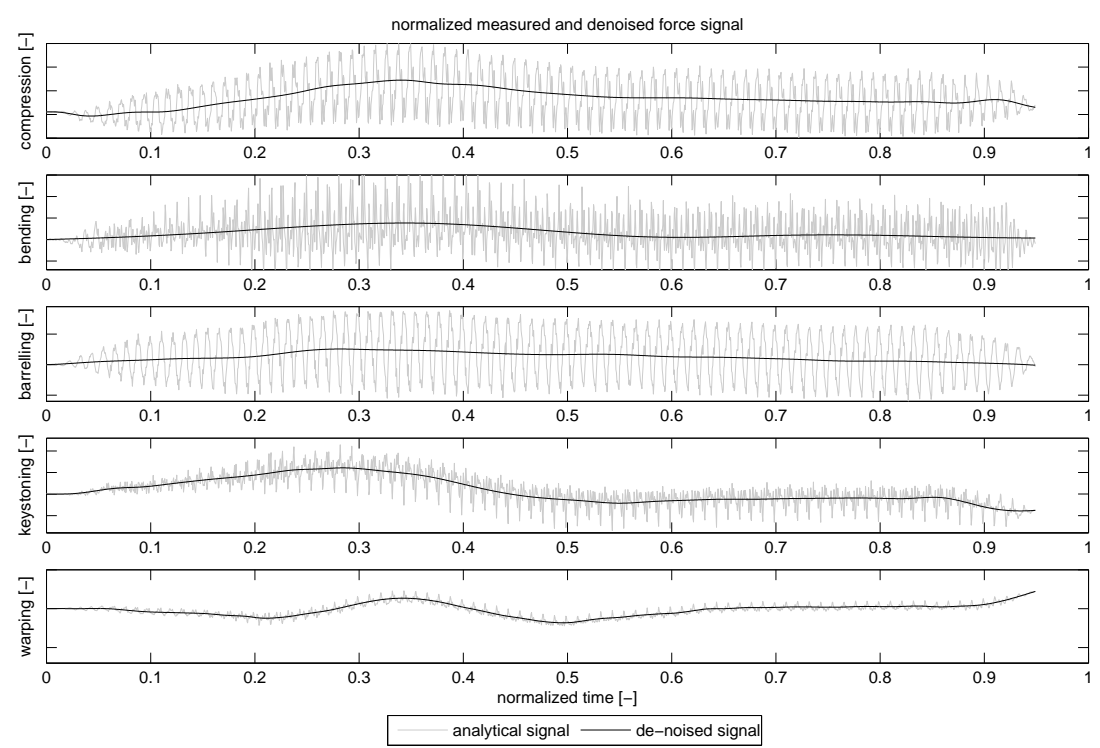

Figure 9: Measured and de-noised signals weld 7.

sisting of IMFs for filtering purposes. The physical approach taken here is in agreement with the energy distribution as found by Flandrin et al. and $\mathrm{Wu}$ and Huang per IMF. The energy in an analytical signal is calculated as

$$
E_{s}=\langle x(t), x(t)\rangle=\int_{-\infty}^{\infty}|x(t)|^{2} \mathrm{~d} t
$$

To de-noise the barrelling signal for weld 7 from Table 2 shown in Fig. 9 it was found that 6 IMFs form the noise and oscillation band in the measured signal and were subsequently subtracted. In Fig. $7 \mathrm{~d}$ it can be seen that after 6 IMFs the energy for the IMFs becomes substantial. The same trend is seen in Fig. 10: in general the lowest number of the IMFs containing the trend of the measured signal can be found were the energy in the IMF increases after a minimum found between IMF $4-7$, consistent with the findings of Flandrin et al. [40, Chapter 3] and Wu and Huang [40, Chapter 5].

\subsection{Feature extraction}

The penultimate step in Fig. 4 is the feature extraction. This work studies the monitoring of the cassette forces during the burn-off phase, which takes place during the reciprocating motion of the moving part, as shown in Fig. 2 and discussed in Section 1.1. It is therefore an unambiguously defined time period during a weld operation. Hence, the start and end times of the burn-off phase can be easily and accurately established from other measurement and control 

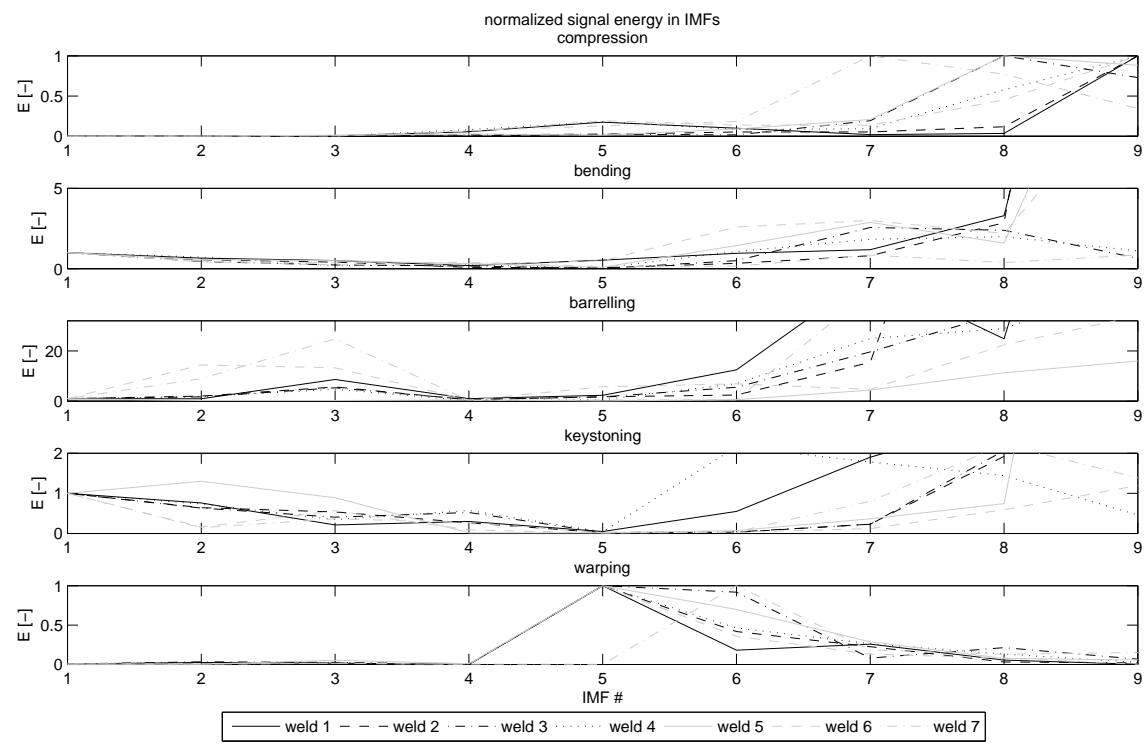

Figure 10: Energy in measure signals per IMF.

signals from the LFW machine. Once these are established, these are then applied to identify and extract the burn-off phase in the measured force signals. As has been done for the practical application of this work. Alternatively, these can be found using standard feature extraction techniques, e.g. using the instantaneous frequency information from the HHT.

\subsection{Experimental Welds}

The results shown in this work are applied to series of preproduction experiments that were conducted on the LF60 linear friction welding machine at the Rolls-Royce plc [4, 41]. These experiments consist of welding two blocks of Ti6Al4V alloy together. The experimental series of welds is shown in Table 2. In this table, seven experimental welds are given. They differ in two ways from each other as is described below.

A difference between the welds are the settings applied to the LFW machine. These settings prescribe the values for the control settings. The control of each of the LFW machine's axes (see Fig. 1 for a definition of the $x, y, z-$ axes) is done with a combination of proportional-integral-derivative (PID) and Amplitude and Phase control (APC) [4]. These seven welds are part of a larger series of test welds. However, it is not necessary to present all these results here. In total 3 settings were used for the APC controller, and 2 different PID values for the control of the tangential $z$-axis, as is shown in Table 2. It is thought that the change of control parameters has a minor influence on the welds, as the normal input parameters such as desired upset and commanded forge force, tangential oscillation frequency etc. were not changed.

Additionally it can be noted that weld 1-4 were used to verify the motion control of the $z$-axis [41]. Weld 5 is made after removing and placing the inner cage back, which always affects the machine's tooling. Weld 6 and 7 come from another batch, as the whole series of welds is made over a longer period of time.

Figure 11a shows all the measured signals, scaled, Fig. 11b shows all the trends of those scaled measured signals. The axes of the plots in this work have been normalized due to Rolls-Royce plc confidentiality. For an appreciation of the machine's dynamics and to understand why it is crucial that advanced signal processing techniques are applied for the welding process monitoring, the typical weld length is a few seconds and generally has over 100 cycles, in addition the four forces acting on the cassette are in the order of 10's of kilonewtons. As mentioned in the introduction, the measured forces on the cassette are related to the deformations as shown in Fig. 3: compression, bending, barrelling, keystoning and warping are all shown in the figures. 


\begin{tabular}{|l|l|l|l|}
\hline weld number & APC Setting & PID settings $z$-axis & weld date \\
\hline \hline 1 & APC1 & PID1 & day 1 \\
2 & APC1 & PID1 & day 1 \\
3 & APC2 & PID1 & day 1 \\
4 & APC2 & PID1 & day 1 \\
5 & APC1 & PID1 & day 160 \\
6 & APC3 & PID2 & day 293 \\
7 & APC3 & PID2 & day 294 \\
\hline
\end{tabular}

Table 2: Weld settings for the Amplitude and Phase Controller (APC) and the Proportional-Integral-Derivative (PID) controller of the linear friction welding machine.

Additionally, for reasons of comparison, after the feature extraction in the time domain, the bending, barrelling, keystoning and warping forces are all started at $t=0 \mathrm{~s}$ and at zero load. However, this did not work for the compression forces, as this made comparison more difficult. These forces have been translated down around zero, using the average value of the 7 welds to align the start value of the compression around zero at $t=t_{b}$.

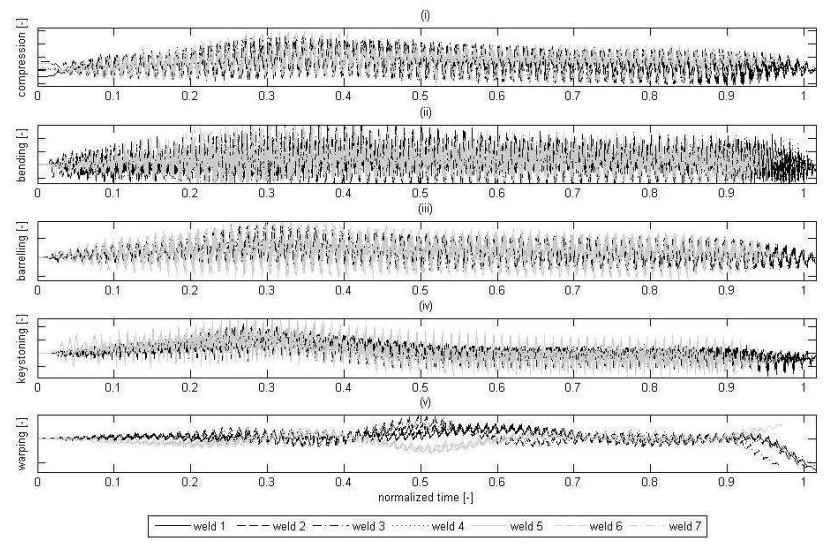

(a)

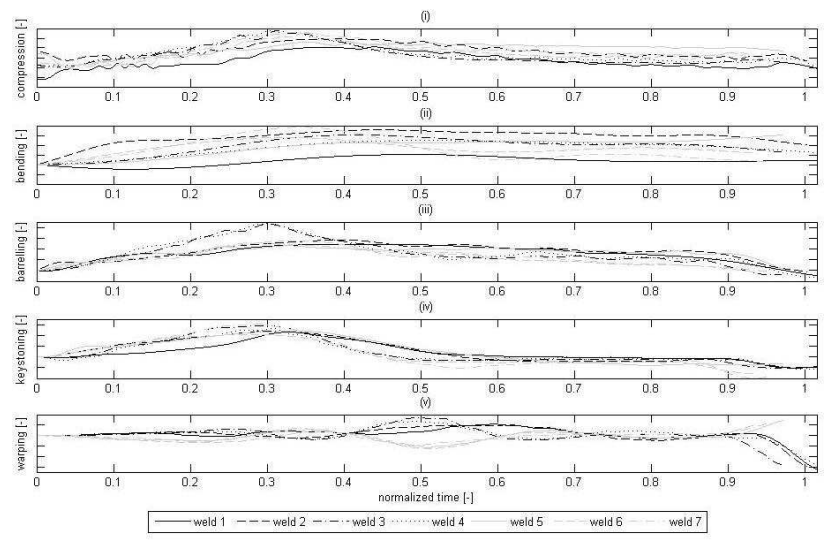

(b)

Figure 11: Measured and de-noised signals(a) Measured signals, (b) de-noised signals for trend monitoring. 


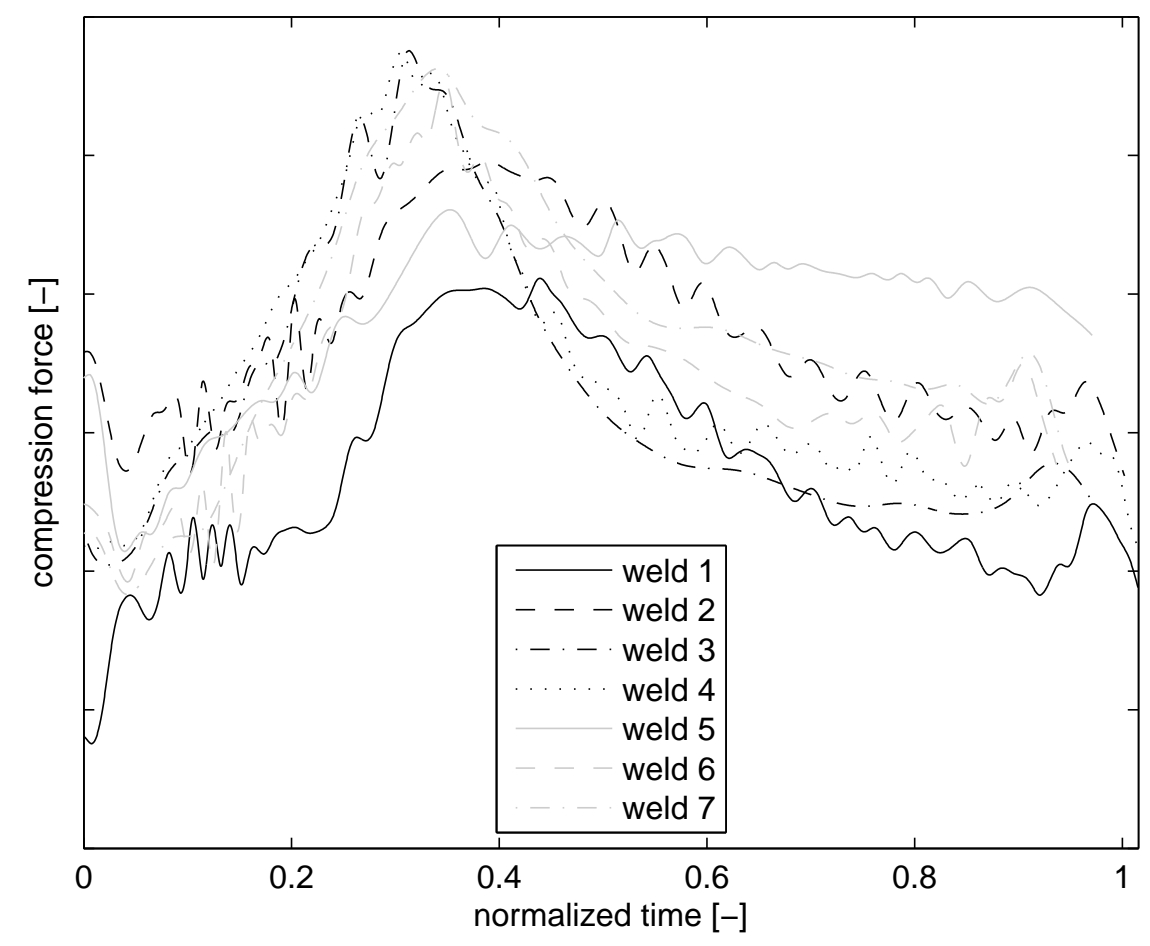

Figure 12: Compression forces on cassette versus time (normalized).

\section{Results}

\subsection{Trends}

\subsubsection{Compression}

The beginning and end effects occurring in the EMD of the compression forces signals resulted in strong deviations at $t=t_{b}$ between measured signals and the trend. Consequently, to approximate the trend, a lower number of IMFs had to be used, resulting in the occurrence of oscillations in the signal, as can be found in Fig. 12. As a result, with the currently applied signal processing, the trends of the compression forces are difficult to compare between each other.

In Fig. 12, it can be seen that different machine settings result in different weld profiles. When comparing these weld with welds with a smaller weld area, the required forces become smaller and the differences become more pronounced, however this would overload this demonstration of using EMD to de-noise cassette forces with too much information. Welds 1,2 and 5 (around $t=0.375$ ) do not have such a high maximum as welds 3, 4, 6 and 7 (around $t=0.31)$.

\subsubsection{Bending}

The bending forces shown in Fig. 13 show similar trends to the compression forces shown in Fig. 12. Note that the trend in weld 1 in Fig. 13 goes below zero. As mentioned above, for reasons of comparison the signal starts at a scaled force of zero at $t=t_{b}$. Therefore, the physical forces still come with a positive sign.

Welds 6 and 7 are relatively similar, but there is no real distinction between the welds 1-4. Also note that the bending force trend for weld 5 increases the whole time.

\subsubsection{Barrelling}

The trends for the barrelling forces are plotted in Fig. 14. For welds 1, 2 and 5 these trends behave in a different manner compared to those of welds 3, 4 and 5, however this behaviour would be more pronounced in case the trends were smooth enough to establish the force rate by differentiating the force with respect to time to study the gradient. It can be seen that weld 1, 2 and 5; weld 3 and 4; and weld 6 and 7 are lying close to each other. 


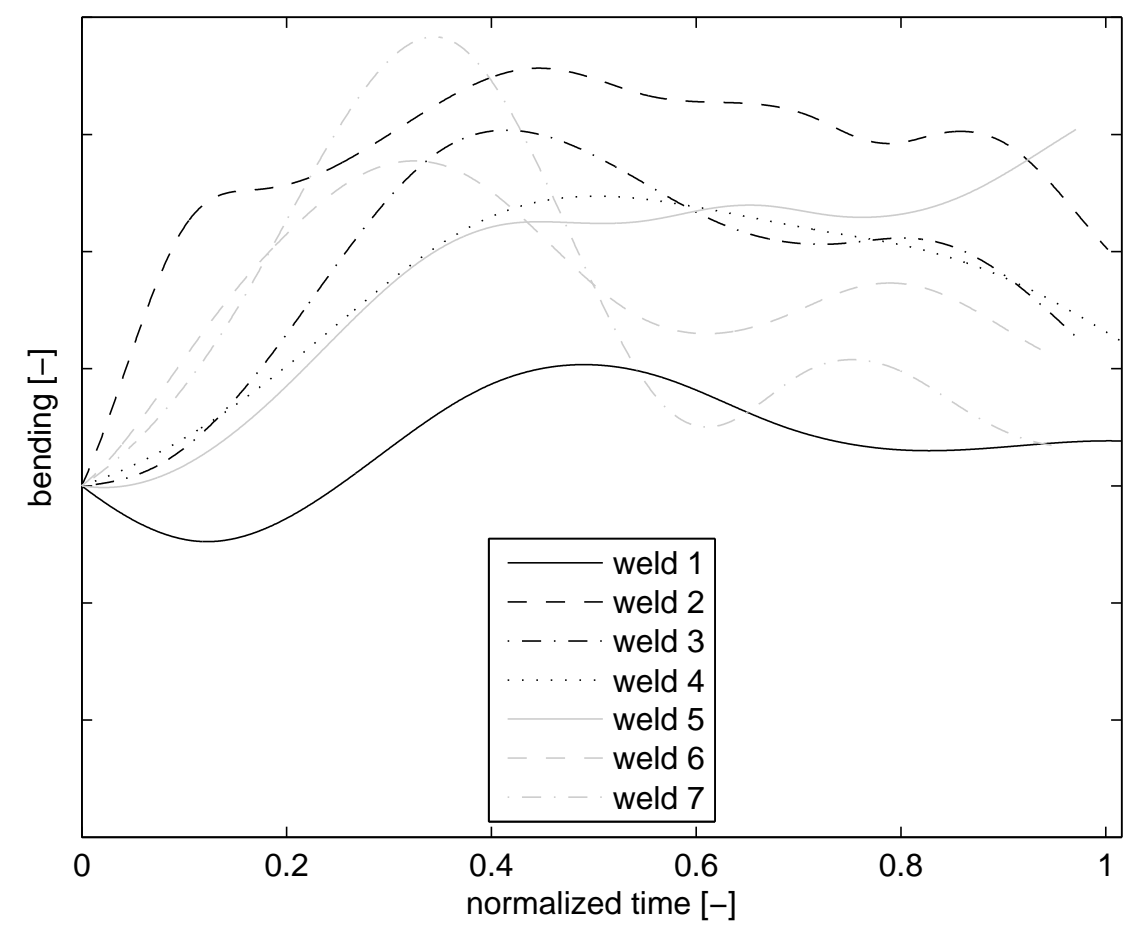

Figure 13: Bending forces on cassette versus time (normalized).

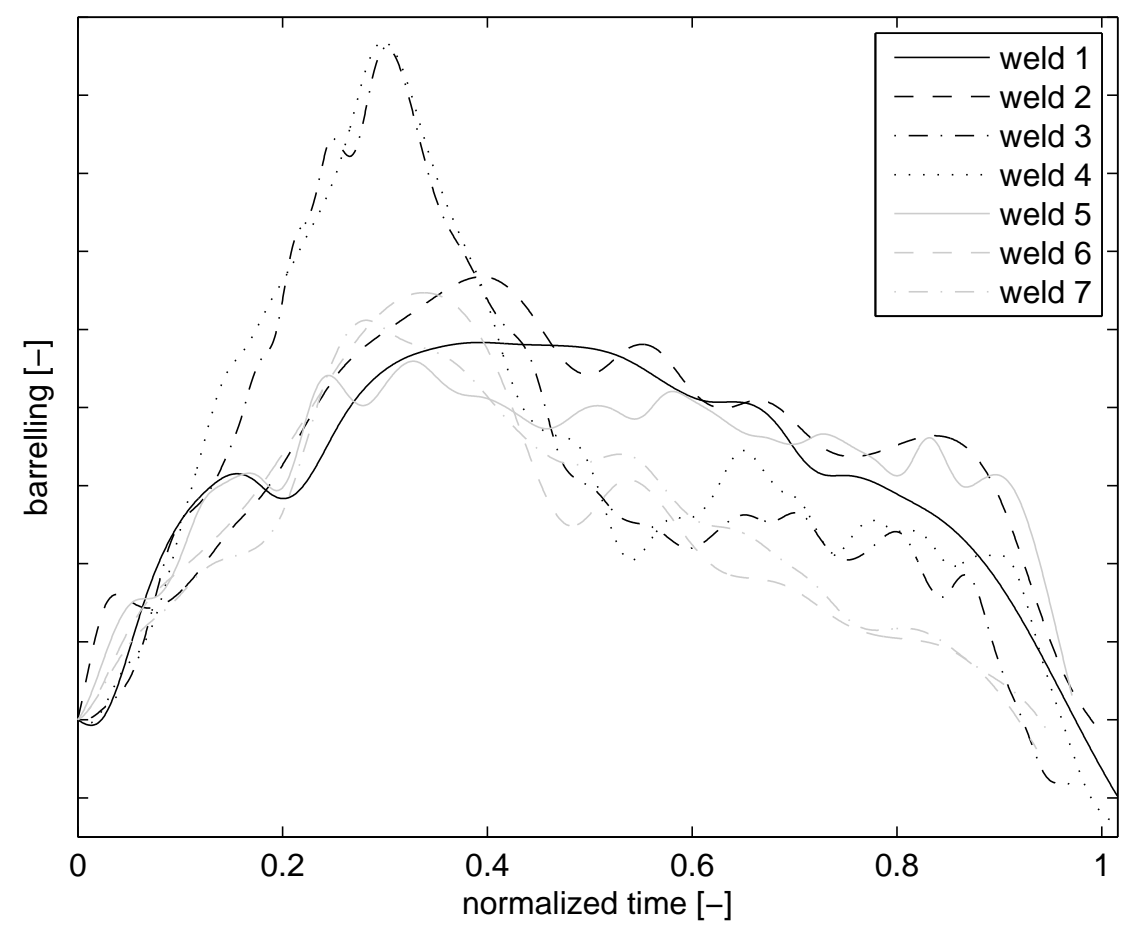

Figure 14: Barrelling forces on cassette versus time (normalized). 


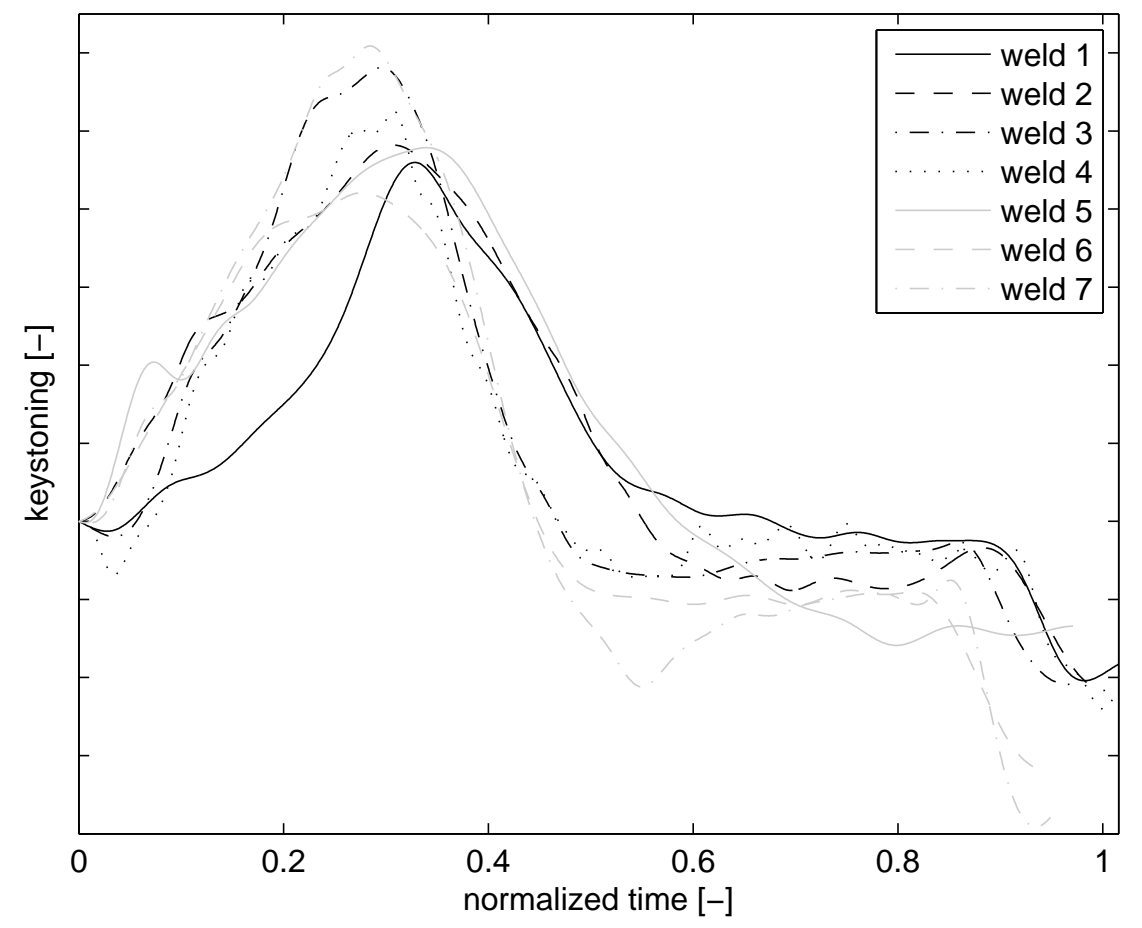

Figure 15: Keystoning forces on cassette versus time (normalized).

\subsubsection{Keystoning}

As can be seen in Fig. 15, the trends in the keystoning forces regardless of weld area size are characterized by a steep positive gradient, leading to a maximum at around $t=0.3$ followed by a steep negative gradient that lasts until $t=0.5-0.55$. From this point onward, the keystone force trends plateau until $t=0.85-0.9$. Note that the trend in weld 5 behaves differently, as the trend keeps a negative gradient in that section. Subsequently, the trend gets a steeper negative gradient again to end with a scaled force. However, welds 1-4 and 6, 7 are not easily distinguishable from each other.

\subsubsection{Warping}

Due to the two different warping directions, the warping forces show the largest variation within the forces studied in this work. The first direction is shown in Fig. 3, the second is a deformation in the opposite direction. Hence in Fig. 16, welds 1-4 show the exact opposite trend of welds 5, 6 and 7. This makes weld 5 quite distinguishable from welds $1-4$, and also welds 6 and 7 are obviously different from welds 1 and 2 and welds 3 and 4 . Weld 1 has an extra maximum occurring at $t=0.375$ compared to the other welds.

\subsection{Analysis of Residue}

\subsubsection{Residue envelope analysis}

From the residues shown in Fig. 17 it can be seen that there is little variation in the magnitude and shapes of the force oscillations of the monitored forces in and amongst the measured sets of the five different forces. The exception here is weld 5, which has quite a different signature compared to the other welds, as was already observed when comparing the trends in the forces. Weld 5 seems not only out of phase with the other welds, the magnitude of the oscillations is different too, which will also shown when analysing the peak values in the power spectral density plots in the next section. 


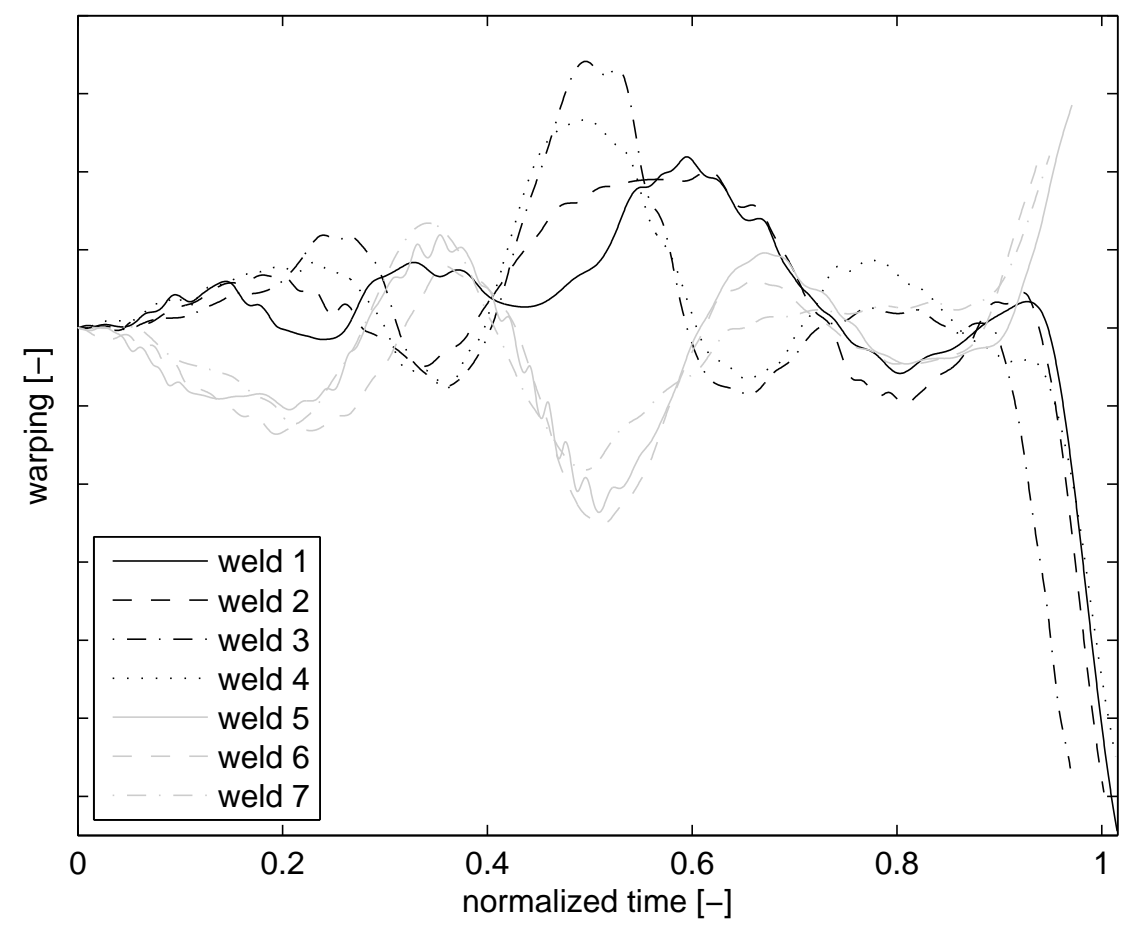

Figure 16: Warping forces on cassette versus time (normalized).

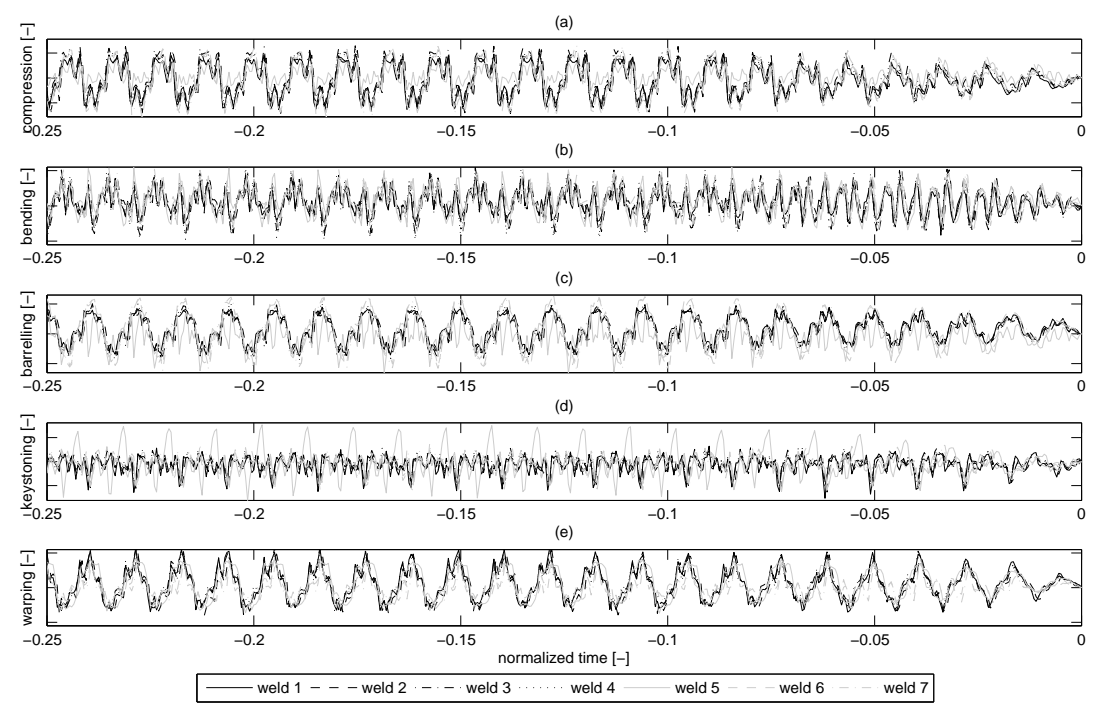

Figure 17: Decay in noise + oscillation aligned at $t=t_{e}$. 


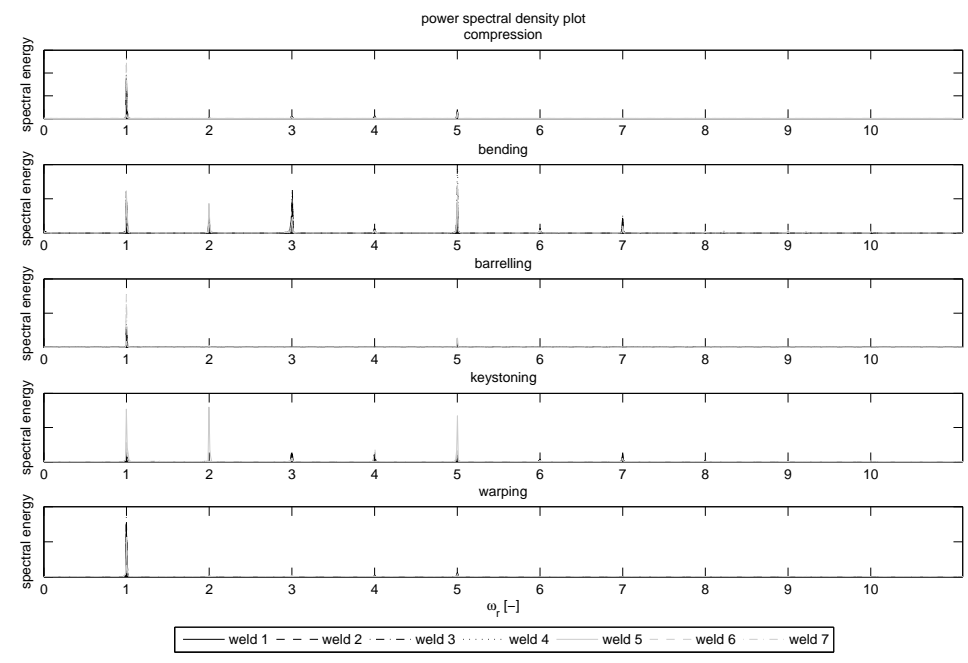

(a)
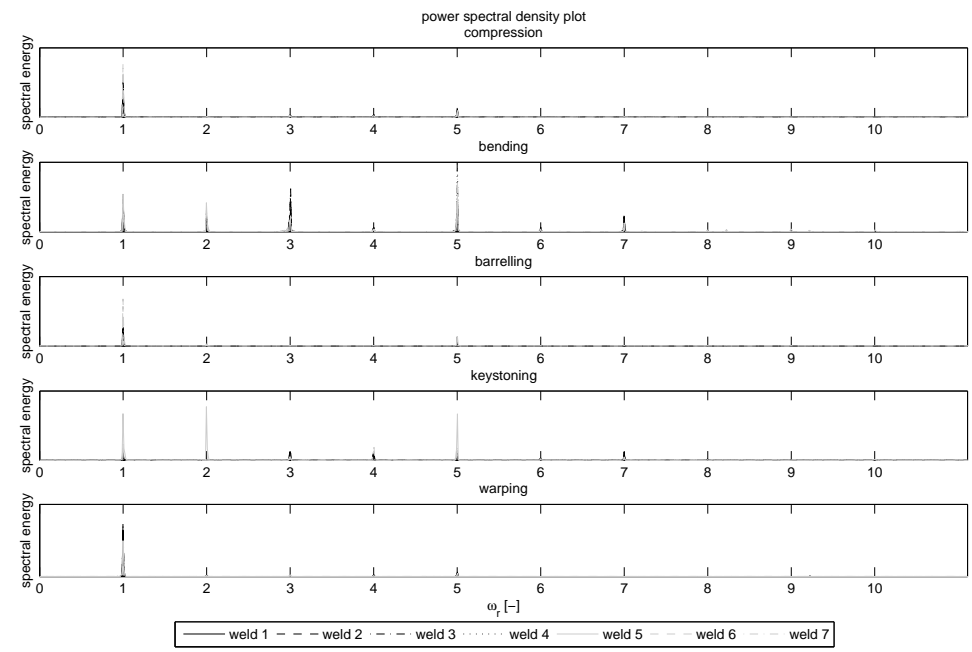

(b)

Figure 18: Power spectral density: (a) power spectral density plot of residue obtained with EMD, (b) power spectral density plot of filtered residue. 


\begin{tabular}{rrrrrrr}
\hline weld 1 & weld 2 & weld 3 & weld 4 & weld 5 & weld 6 & weld 7 \\
\hline$-12.6 \%$ & $-12.5 \%$ & $-12.5 \%$ & $-12.6 \%$ & $-0.4 \%$ & $-12.5 \%$ & $-12.6 \%$ \\
$-0.4 \%$ & $-0.4 \%$ & $-0.4 \%$ & $-0.4 \%$ & $-12.5 \%$ & $-0.4 \%$ & $-0.4 \%$ \\
$-0.7 \%$ & $-0.7 \%$ & $-1.2 \%$ & $-1.4 \%$ & $-1.5 \%$ & $-0.7 \%$ & $-0.7 \%$ \\
\hline \multicolumn{7}{c}{ bending } \\
\hline$-12.9 \%$ & N/A & $-0.4 \%$ & $-0.4 \%$ & $-12.6 \%$ & $-0.4 \%$ & $-0.4 \%$ \\
$-0.4 \%$ & N/A & $-1.3 \%$ & $-1.3 \%$ & $-0.4 \%$ & $-12.7 \%$ & $-12.6 \%$ \\
$-1.3 \%$ & $-1.4 \%$ & $-12.6 \%$ & $-12.6 \%$ & $-3.2 \%$ & $-3.1 \%$ & $-1.4 \%$ \\
\hline \multicolumn{7}{c}{ barrelling } \\
\hline$-12.6 \%$ & $-12.6 \%$ & $-12.6 \%$ & $-12.6 \%$ & $-0.4 \%$ & $-12.6 \%$ & $-12.6 \%$ \\
$-0.4 \%$ & $-0.4 \%$ & $-0.4 \%$ & $-0.4 \%$ & $-12.6 \%$ & N/A & $-13.3 \%$ \\
$-0.7 \%$ & $-3.3 \%$ & $-3.3 \%$ & $-0.7 \%$ & $-3.2 \%$ & $-2.9 \%$ & $-3.2 \%$ \\
\hline \multicolumn{7}{c}{ keystoning } \\
\hline$-1.4 \%$ & $-12.6 \%$ & $-12.6 \%$ & $-12.6 \%$ & $-3.3 \%$ & $-12.7 \%$ & $-12.7 \%$ \\
$-0.1 \%$ & $-0.7 \%$ & $-0.7 \%$ & $-0.7 \%$ & $-12.6 \%$ & $-0.7 \%$ & $-0.4 \%$ \\
$-12.7 \%$ & $-1.4 \%$ & $-1.4 \%$ & $-0.1 \%$ & $-0.4 \%$ & $-0.4 \%$ & $-0.7 \%$ \\
\hline \multicolumn{7}{c}{ warping } \\
\hline$-12.5 \%$ & $-12.6 \%$ & $-12.6 \%$ & $-12.5 \%$ & $-12.2 \%$ & $-12.5 \%$ & $-12.7 \%$ \\
$-0.4 \%$ & $-0.3 \%$ & $-0.4 \%$ & $-0.4 \%$ & $-1.4 \%$ & $-0.4 \%$ & $-0.4 \%$ \\
$-0.7 \%$ & $-0.9 \%$ & $-12.9 \%$ & $-0.7 \%$ & $-0.7 \%$ & $-3.2 \%$ & $-3.3 \%$ \\
\hline 7
\end{tabular}

Table 3: Relative differences between the amplitudes of the three largest peaks in the frequency spectrum of each force after using EMD as a filter bank and filtering in a power spectral density analysis.

\subsubsection{Frequency domain analysis}

In this section the frequency content of the residue formed by the IMFs that span the noise and oscillation band of the measured signal is studied. This allows for the detection of undesired frequency components in the force signals. In Fig. 18, the power spectral density of the residue is shown calculated with the Fast Fourier Transform.

Firstly, in the figure, it can be noted that the excitation frequency, i.e. the frequency $\omega_{\mathrm{t}}$ of the reciprocating motion in $z$-direction is dominant, which is as expected. In the figure, the frequency axis is scaled such that a frequency when $\omega_{\mathrm{r}}=1, \omega_{\mathrm{r}}=\omega_{\mathrm{t}}$. Higher harmonics of $\omega_{\mathrm{t}}$ are clearly visible. Furthermore, the power of the signal components in the frequency domain for the compression and warping forces is of one order higher than those of the bending, barrelling and keystoning forces. Additionally, the power of the components in welds 1 and 2, welds 3 and 4 , and welds 6 and 7 is relatively similar in magnitude within these little group of welds, the three groups themselves are dissimilar in magnitude. The magnitudes of weld 5 do not match up with those of welds 1 and 2 . This means that analyzing the average amplitude of the residue can help to determine whether a weld belongs to a certain group. The more subtle power spectra only constitute a small contribution in the total power in the signal. These contributions are so small, that even when considering that the power in a oscillating signal is proportional to the square of the oscillation amplitude, the amplitude of the reciprocating frequency and its higher order harmonics are still more than one or two orders larger than the amplitudes of the other frequency components.

The residue obtained with EMD de-noising is given in Fig. 18a and the residue established with filtering using a standard first-order filter is shown in Fig. 18b. To compare these, the same scale is used. When zooming in to look at the more subtle contributions, comparing Fig. 18a and 18b with each other, the same subtle contributions are clearly visible. Furthermore, the residue established by de-noising the measured signal with EMD, shows some content for near zero frequencies. Because of the beginning and end effects occurring in standard EMD, a small deviation can occur below the deviation threshold as discussed in Section 2.2.3, where the algorithm shown in Fig. 8 is explained. This small deviation locally adds a small constant value mostly at the beginning of the burn-off period, which explains the near zero frequency contributions.

There is some more content when $\omega_{\mathrm{r}}<1$ for the compression residue, which possibly indicates mode mixing, 
however, this contribution is relatively small. Both EMD de-noised and filtered residuals of the warping forces show some more low frequency $\left(\omega_{\mathrm{r}}<1\right)$ content.

A more quantitative comparison of the two power spectral density analyses is made in Table 3 . Here the amplitude of the three largest peaks of the power components in the signal in the frequency spectrum are compared:

$$
\varepsilon \%=\frac{P_{\mathrm{EMD}}-P_{\mathrm{FO}-\text { filter }}}{P_{\mathrm{EMD}}} \times 100 \%,
$$

where $P_{\mathrm{EMD}}$ is the amplitude of the power component in the residue obtained by de-noising using EMD, and $P_{\mathrm{FO}-\text { filter }}$ is the amplitude of the power component at the same frequency of the residue obtained with filtering using a standard first-order filter. Where the entry says "N/A" in Table 3 this means that the highest peaks coming from the filtering belong to different higher order harmonics of the reciprocating motion's frequency. It is clearly visible that amplitudes of signal components in the frequency domain are consistently smaller than those obtain using the EMD-based denoising method. This means that the amplitudes of the filtered residues are smaller than those of the EMD-based de-noised residues, meaning that the noise is removed a further when applying the EMD-based de-noising approach. This reinforces the choice of using EMD-based de-noising in order to de-noise the force signals in the linear friction welding process. Using the well-known approximation $\sqrt{1+2 \epsilon} \approx 1+\epsilon$, the percentage difference when considering the amplitude rather than the power of the signal components, for small $\epsilon$ would be about a factor 2 smaller, and should be multiplied by the correction for the wave shape.

\subsection{Overall comparison of the welds}

Due to the fact that the inner cage was taken out and placed back in the linear friction welding machine, weld 5 shows a different signature for the force profiles than the other welds, this is because the tooling needs to settle during the first weld after taking out the inner cage. Subsequent welds will show a larger repeatability.

The compression and warping forces and the power spectral density analyses show that different welds settings for welds 1 and 2, welds 3 and 4 and welds 6 and 7 give slightly different results and allow these welds to be grouped together. Overall, as could be expected, the welds show similarity. However, the fact that this can be studied means that obtaining the trends in the forces can be applied to monitor the repeatability of the welds.

\section{Conclusions}

This work studies the development of a cassette forces monitoring system for a linear friction welding machine. The monitoring of the forces consists mainly of an analysis for the trend in the signal during the period, when the part to be welded on the substrate is undergoing an oscillatory motion, i.e. during the burn-off period. To obtain the trend in the signal, the following method has been established:

1. The data is captured from the linear friction welding (LFW) machine using its sensory and data storage systems.

2. The data is de-noised before other data processing takes place, to prevent the adverse effects on accuracy by beginning and end effects. For robust data processing, a de-noising strategy to find the trends in the signals based on empirical mode decomposition (EMD) has been designed using the intrinsic mode functions (IMFs) as filter bank.

3. The selection of the number of IMFs that form the noise and oscillation band is automated and is based on the average frequency of the IMFs during the burn-off period, but can also automatically adapt to a lower number of IMFs when the trend and the measured signal differ too much at beginning and end of the burn-off period.

4. The feature extraction takes place in the time domain, where begin and end times of the burn-off phase are identified from the sensory system of the LFW machine, and the forces acting on the cassette are extracted from their respective vectors utilizing the begin and end times.

5. The data are compared against previously analyzed welds established with the outlined methodology.

Some other contributions established in this work are the following:

- The methodology has been demonstrated with the analysis of test welds performed on a LFW machine. 
- The automated selection of the IMFs that form noise and process induced oscillation band has been based on the average instantaneous frequency of the IMFs during the burn-off phase.

- For frequency domain analysis, the EMD de-noising strategy has been benchmarked against traditional filtering.

- Applying EMD based de-noising of the signal gives more accurate information on the trend in the signal.

\section{Acknowledgements}

The authors wish to acknowledge the support of the European Commission through the 7th Framework Programme under call FP7-AAT-2007-RTD-1 (FLEXA; grant agreement 213734) and support of EPSRC through SAMULET Project 5: Processing Advanced Materials.

\section{References}

[1] R. Turner, J.-C. Gebelin, R. M. Ward, R. C. Reed, Linear friction welding of Ti-6Al-4V: Modelling and validation, Acta Mater. 59 (10) (2011) 3792-3803.

[2] R. D. Trask, Apparatus and method for friction welding, US 6688512 B2, patent (February 2004).

[3] I. Bhamji, M. Preuss, P. L. Threadgill, A. C. Addison, Solid state joining of metals by linear friction welding: A literature review, Mater. Sci. Technol. 27 (1) (2011) 2-12.

[4] D. T. Williams, A. R. Plummer, P. Wilson, Fault detection of a linear friction welding production system using an analytical model, in: R. Raimund Ege, L. Koszalka (Eds.), Proceedings of the Eighth International Conference on Systems 2013, IARIA, IARIA XPS Press, Sevilla, 2013, pp. 69-76.

[5] A. Vairis, M. Frost, High frequency linear friction welding of a titanium alloy, Wear 217 (1) (1998) 117-131.

[6] R. A. Hameed, M. A. Mannan, A. Y. C. Nee, The cutting force measurement in a fixturing setup with instrumented locators, Int. J. Adv. Manuf. Technol. 23 (11-12) (2004) 783-793.

[7] J. V. Abellan-Nebot, F. R. Subirón, A review of machining monitoring systems based on artificial intelligence process models, Int. J. Adv. Manuf. Technol. 47 (1-4) (2010) 237-257.

[8] P. G. Benardos, G.-C. Vosniakos, Predicting surface roughness in machining: A review, Int. J. Mach. Tools Manuf. 43 (8) (2003) 833-844.

[9] S. Y. Liang, R. L. Hecker, R. G. Landers, Machining process monitoring and control: The state-of-the-art, Trans. ASME-J. Manuf. Sci Eng. 126 (2) (2004) 297-310.

[10] J. T. Roth, D. Djurdjanovic, X. Yang, L. Mears, T. Kurfess, Quality and inspection of machining operations: Tool condition monitoring, Trans. ASME-J. Manuf. Sci Eng. 132 (4) (2010) 041015-1-041015-16.

[11] R. Teti, K. Jemielniak, G. O’Donnell, D. Dornfeld, Advanced monitoring of machining operations, CIRP Ann.-Manuf. Technol. 59 (2) (2010) 717-739.

[12] I. N. Tansel, B. L. Grisso, G. Singh, G. Singh, S. Korla, R. Duran, L. W. Salvino, Wear estimation by testing the elastic behavior of tool surface, Int. J. Mach. Tools Manuf. 51 (10-11) (2011) 745-752.

[13] C. F. Bisu, M. Zapciu, O. Cahuc, A. Gérard, M. Anica, Envelope dynamic analysis: a new approach for milling process monitoring, Int. J. Adv. Manuf. Technol. 62 (5-8) (2012) 471-486.

[14] T. Kalvoda, Y.-R. Hwang, A cutter tool monitoring in machining process using Hilbert-Huang transform, Int. J. Mach. Tools Manuf. 50 (5) (2010) 495-501.

[15] H. Shao, X. Shi, L. Li, Power signal separation in milling process based on wavelet transform and independent component analysis, Int. J. Mach. Tools Manuf. 51 (9) (2011) 701-710.

[16] B. Chen, X. Chen, B. Li, Z. He, H. Cao, G. Cai, Reliability estimation for cutting tools based on logistic regression model using vibration signals, Mech. Syst. Signal Process. 25 (7) (2011) 2526-2537.

[17] P. S. Sivasakthivel, V. Velmurugan, R. Sudhakaran, Prediction of vibration amplitude from machining parameters by response surface methodology in end milling, Int. J. Adv. Manuf. Technol. 53 (5-8) (2011) 453-461.

[18] C.-C. Chen, N.-M. Liu, K.-T. Chiang, H.-L. Chen, Experimental investigation of tool vibration and surface roughness in the precision endmilling process using the singular spectrum analysis, Int. J. Adv. Manuf. Technol. 63 (5-8) (2012) 797-815.

[19] Z. Yang, Z. Yu, Grinding wheel wear monitoring based on wavelet analysis and support vector machine, Int. J. Adv. Manuf. Technol. 62 (1-4) (2012) 107-121.

[20] T. Boutros, M. Liang, Detection and diagnosis of bearing and cutting tool faults using hidden Markov models, Mech. Syst. Signal Process. 25 (6) (2011) 2102-2124.

[21] B. Kilundu, P. Dehombreux, X. Chiementin, Tool wear monitoring by machine learning techniques and singular spectrum analysis, Mech. Syst. Signal Process. 25 (1) (2011) 400-415.

[22] D. A. Tobon-Mejia, K. Medjaher, N. Zerhouni, CNC machine tool's wear diagnostic and prognostic by using dynamic Bayesian networks, Mech. Syst. Signal Process. 28 (2012) 167-182.

[23] C. L. Yen, M. C. Lu, J. L. Chen, Applying the self-organization feature map (SOM) algorithm to AE-based tool wear monitoring in microcutting, Mech. Syst. Signal Process. 34 (1-2) (2013) 2102-2124.

[24] D. Trimble, J. Monaghan, G. E. O’Donnell, Force generation during friction stir welding of AA2024-T3, CIRP Ann.-Manuf. Technol. 61 (2012) 9-12. 
[25] K. J. Kalinski, M. A. Galewski, Chatter vibration surveillance by the optimal-linear spindle speed control, Mech. Syst. Signal Process. 25 (1) (2011) 383-399.

[26] Y. Zhou, T. Tao, X. Mei, G. Jiang, N. Sun, Feed-axis gearbox condition monitoring using built-in position sensors and EEMD method, Robot. Comput.-Integr. Manuf. 27 (4) (2011) 785-793.

[27] D. Pérez-Canales, J. Álvarez Ramírez, J. C. Jáuregui-Correa, L. Vela-Martínez, G. Herrera-Ruiz, Identification of dynamic instabilities in machining process using the approximate entropy method, Int. J. Mach. Tools Manuf. 51 (6) (2011) 556-564.

[28] J. Jin, J. Shi, Automatic feature extraction of waveform signals for in-process diagnostic performance improvement, J. of Intell. Manuf. 12 (3) (2001) 257-268.

[29] D. M. Longanbach, T. R. Kurfess, In-process gage frequency response measurement, Mechatronics 11 (6) (2001) $745-757$.

[30] A. Rabani, I. Marinescu, D. Axinte, Acoustic emission energy transfer rate: A method for monitoring abrasive waterjet milling, J. Sound Vib. 61 (2012) 80-89.

[31] L. X. Kong, S. Nahavandi, On-line tool condition monitoring and control system in forging process, J. Mater. Process. Technol. 125-126 (2002) 464-470.

[32] N. E. Huang, S. R. Shen, Z. Long, M. C. Wu, H. H. Shih, Q. Zheng, N.-C. Yen, C. C. Tung, H. H. Liu, The empirical mode decomposition and the Hilbert spectrum for nonlinear and non-stationary time series analysis, Proc.R.Soc. A 454 (1971) (1998) 903-995.

[33] Z. Wu, N. E. Huang, Ensemble empirical mode decomposition: A noise-assisted data analysis method, Adv.Adap. Data Anal. 1 (1) (2009) $1-41$.

[34] Z. K. Peng, P. W. Tse, F. L. Chu, An improved Hilbert-Huang transform and its application in vibration signal analysis, J. Sound Vib. 286 (1-2) (2005) 187-205.

[35] N. Roveri, A. Carcaterra, Damage detection in structures under traveling loads by Hilbert-Huang transform, Mech. Syst. Signal Process. 28 (2012) 128-144.

[36] X. Zhao, T. H. Patel, M. J. Zuo, Multivariate EMD and full spectrum based condition monitoring for rotating machinery, Mech. Syst. Signal Process. 27 (2012) 712-728.

[37] L.-S. Law, J. H. Kim, W. Y. H. Liew, S.-K. Lee, An approach based on wavelet packet decomposition and Hilbert-Huang transform (WPDHHT) for spindle bearings condition monitoring, Mech. Syst. Signal Process. 33 (2012) 197-211.

[38] E. Kreyszig, Introductory Functional Analysis with Applications, John Wiley \& Sons, New York, 1978.

[39] A. Tan, Mathworks MATLAB ${ }^{\circledR}$ Central File Exchange: Hilbert-Huang Transform, The Mathworks Inc., website, accessed on 29-05-2013, URL: http://www.mathworks.co.uk/matlabcentral/fileexchange/19681-hilbert-huang-transform (2008).

[40] N. E. Huang, S. S. P. Shen (Eds.), Hilbert-Huang Transform and Its Applications, Interdisciplinary Mathematical Sciences, World Scientific, Singapore, 2005.

[41] D. T. Williams, P. Wilson, A. R. Plummer, Dynamic modelling of a linear friction welding machine actuation system, in: D. N. Johnston, A. R. Plummer (Eds.), Proceedings of the PTMC/ASME Symposium on Fluid Power and Motion Control, PTMC, PTMC, Bath, 2012, pp. 379-396. 